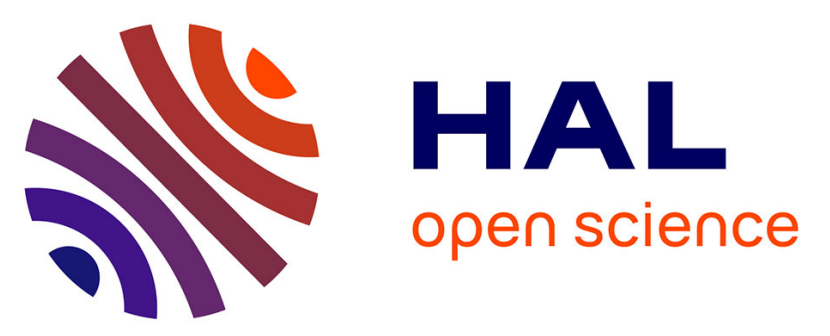

\title{
Suprathermal electrons near the nucleus of comet 67P/Churyumov-Gerasimenko at 3 AU: Model comparisons with Rosetta data
}

H. Madanian, T. E. Cravens, A. Rahmati, R. Goldstein, J. Burch, A. I. Eriksson, N. J. T. Edberg, Pierre Henri, K. Mandt, G. Clark, et al.

\section{To cite this version:}

H. Madanian, T. E. Cravens, A. Rahmati, R. Goldstein, J. Burch, et al.. Suprathermal electrons near the nucleus of comet $67 \mathrm{P} /$ Churyumov-Gerasimenko at $3 \mathrm{AU}$ : Model comparisons with Rosetta data. Journal of Geophysical Research Space Physics, 2016, 121 (6), pp.5815-5836. 10.1002/2016JA022610 . insu-01352458

\section{HAL Id: insu-01352458 \\ https://hal-insu.archives-ouvertes.fr/insu-01352458}

Submitted on 25 Oct 2016

HAL is a multi-disciplinary open access archive for the deposit and dissemination of scientific research documents, whether they are published or not. The documents may come from teaching and research institutions in France or abroad, or from public or private research centers.
L'archive ouverte pluridisciplinaire HAL, est destinée au dépôt et à la diffusion de documents scientifiques de niveau recherche, publiés ou non, émanant des établissements d'enseignement et de recherche français ou étrangers, des laboratoires publics ou privés. 


\section{Journal of Geophysical Research: Space Physics}

\section{RESEARCH ARTICLE \\ 10.1002/2016JA022610 \\ Key Points: \\ - Plasma environment around comet \\ Suprathermal electrons near the nucleus of comet 67P/Churyumov-Gerasimenko at $3 \mathrm{AU}$ : Model comparisons with Rosetta data} $67 \mathrm{P}$ at $3 \mathrm{AU}$

- Rosetta spacecraft electron density measurements in the coma and solar wind

- Relation between hot and cold electron populations at different distances to the nucleus

Correspondence to:

T. E. Cravens,

cravens@ku.edu

\section{Citation:}

Madanian, H., et al. (2016), Suprathermal electrons near the nucleus of comet 67P/Churyumov-Gerasimenko at $3 \mathrm{AU}$ : Model comparisons with Rosetta data, J. Geophys. Res. Space Physics, 121, 5815-5836, doi:10.1002/2016JA022610.

Received 29 FEB 2016 Accepted 3 JUN 2016

Accepted article online 7 JUN 2016 Published online 28 JUN 2016

\author{
H. Madanian ${ }^{1}$, T. E. Cravens ${ }^{1}$, A. Rahmati' ${ }^{1}$, R. Goldstein ${ }^{2}$, J. Burch' ${ }^{2}$, A. I. Eriksson ${ }^{3}$, N. J. T. Edberg ${ }^{3}$, \\ P. Henri ${ }^{4}$, K. Mandt ${ }^{2}$, G. Clark ${ }^{5}$, M. Rubin ${ }^{6}$, T. Broiles ${ }^{2}$, and N. L. Reedy ${ }^{1}$ \\ ${ }^{1}$ Department of Physics and Astronomy, University of Kansas, Lawrence, KS, USA, ${ }^{2}$ Southwest Research Institute, San \\ Antonio, Texas, USA, ${ }^{3}$ Swedish Institute of Space Physics, Uppsala, Sweden, ${ }^{4}$ Laboratoire de Physique et Chimie de \\ I'Environnement et de l'Espace CNRS, Orléans, France, ${ }^{5}$ Johns Hopkins University Applied Physics Laboratory, Laurel, \\ Maryland, USA, ${ }^{6}$ Physikalisches Institut, University of Bern, Bern, Switzerland
}

Abstract Observations of the coma near the nucleus of comet 67P/Churyumov-Gerasimenko (67P) made by the IES (Ion and Electron Sensor) instrument onboard the Rosetta Orbiter during late 2014 showed that electron fluxes greatly exceeded solar wind electron fluxes. The IES is part of the Rosetta Plasma Consortium. This paper reports on electron energy spectra measured by IES near the nucleus as well as approximate densities and average energies for the suprathermal electrons when the comet was at a heliocentric distance of about $3 \mathrm{AU}$. Comparisons are made with electron densities measured by other instruments. The high electron densities observed (e.g., $n_{e} \approx 10-100 \mathrm{~cm}^{-3}$ ) must be associated with the cometary ion density enhancement created mainly by the photoionization of cometary gas by solar radiation; there are other processes that also contribute. Quasineutrality requires that the electron and ion densities be the same, and under certain conditions an ambipolar electric field is required to achieve quasi-neutrality. We present the results of a test particle model of cometary ion pickup by the solar wind and a two-stream electron transport code and use these results to interpret the IES data. We also estimate the effects on the electron spectrum of a compression of the electron fluid parcel. The electrons detected by IES can have energies as high as about 100-200 eV near the comet on some occasions, in which case the hot electrons can significantly enhance ionization rates of neutrals via impact ionization.

\section{Introduction}

The Rosetta spacecraft arrived at comet 67P/Churyumov-Gerasimenko (or 67P) in August 2014, and during late 2014 it orbited the nucleus at radial distances of about $10-100 \mathrm{~km}$. The comet (and spacecraft) during this time period was located about $3 \mathrm{AU}$ from the Sun when the cometary activity was still low. The ROSINA (Rosetta Orbiter Spectrometer for lon and Neutral Analysis) COPS (Comet Pressure Sensor) sensor [Balsiger et al., 2007] measured neutral densities at these cometocentric distances of $n_{n} \approx 10^{7} \mathrm{~cm}^{-3}$, consistent with a gas production rate of $Q \approx 10^{26} \mathrm{~s}^{-1}$ [Bieler et al., 2015], which is more than 7000 times less than comet Halley's gas production rate during its perihelion passage in 1986.

Previous missions to comets were limited to single flybys, allowing just a "snapshot" of a cometary environment at specific cometary and heliocentric distances, as the spacecraft crossed different plasma boundaries formed near the comets. Flybys of comets 1P/Halley, 21P/Giacobini-Zinner, 26P/Grigg-Skjellerup, and 19P/Borrelly by the ICE, Giotto, VEGA, Suisei, Sakigake, and Deep Space 1 spacecraft all occurred when the comets were near their perihelia, and the production rates of dust and neutrals were much higher $(\approx 1000$ times) than for the comet 67P at $3 \mathrm{AU}$ considered here. For instance, the ion composition and dynamics at comet Halley was studied using Giotto's ion mass spectrometer [e.g., Balsiger et al., 1986], and a significant deflection and slowing of solar wind due to the pickup ion mass loading process was observed. Pickup ions are ions created by ionization of relatively slow neutral species and then subject to the Lorentz force associated with the solar wind motional electric field and the interplanetary magnetic field. These freshly created ions then move, or partially move, with the solar wind. Water group pickup ions were seen outside the bow shock of comet GiacobiniZinner by the ULECA (Ultra Low-Energy Charge Analyzer) instrument on ICE (International Cometary Explorer) [Gloeckler et al., 1986] and by the Giotto ion mass spectrometer [Neugebauer et al., 1987], and Cravens $[1986,1989]$ carried out test particle calculations of these pickup ions, using uniform background fields. 
The Rosetta spacecraft carries a full suite of plasma instruments in order to carry out long-term observations of the plasma environment of comet 67P. The Rosetta Plasma Consortium (RPC) [Carr et al., 2007] is a package of five plasma and field instruments, including the lon and Electron Sensor (IES) [Burch et al., 2007], the lon Composition Analyzer (ICA) [Nilsson et al., 2007], the Langmuir Probe (LAP) [Eriksson et al., 2007], the Mutual Impedance Probe (MIP) [Trotignon et al., 2007], and the Magnetometer (MAG) [Glassmeier et al., 2007]. Collective study of the measurements made by the RPC instruments is providing a wealth of information on plasma processes at different stages of the interaction between the solar wind and the comet. On 7 August 2014, when Rosetta was at a distance of $100 \mathrm{~km}$ from the comet, the ICA instrument started to detect water group ions originating from the ionization of the cometary neutral environment [Nilsson et al., 2015]. The outgassing rate of the comet at that time was estimated to be around $10^{26} \mathrm{~s}^{-1}$. For such a production rate, a weak interaction between the solar wind and the cometary neutral coma was predicted [Koenders et al., 2015; Rubin et al., 2014; Hansen et al., 2007]. Later on 21 September 2014, the solar wind protons detected by ICA were found to be deflected from the sunward direction by about $20-30^{\circ}$, and this was attributed to conservation of momentum associated with the production of cometary pickup ions [Nilsson et al., 2015]. Water group ions with energies up to $\sim 1 \mathrm{keV}$ were observed by ICA in a direction opposite to the deflection of the solar wind, as predicted by conservation of momentum.

Measurements of electron densities by LAP and MIP near comet 67P showed values that were between about 40 and $200 \mathrm{~cm}^{-3}$ with an average variation that goes inversely as cometocentric distance $r$, albeit with a large variance due to variations in comet latitude and longitude [Edberg et al., 2015]. Furthermore, Odelstad et al. [2015] demonstrated a good correlation between the electron density and the neutral density measured by ROSINA/COPS (Rosetta Orbiter Spectrometer for Ion and Neutral Analysis, and Comet Pressure Sensor, respectively [Balsiger et al., 2007; Bieler et al., 2015].

Early IES results near the comet were reported by Burch et al. [2015], Goldstein et al. [2015], Broiles et al. [2015], and Clark et al. [2015]. The trajectories of solar wind protons and alpha particles exhibit modest deflections (several degrees) as well as some attenuation (30\%) in density due to charge exchange collisions with cometary neutrals. Both the IES and the ICA parts of the RPC [Nilsson et al., 2015] showed both deflected protons and pickup cometary ions with energies ranging from a few $\mathrm{eV}$ up to somewhat less than $1 \mathrm{keV}$, which is much less than the energies expected for fully picked-up ions. Goldstein et al. [2015] have also reported on pickup ions with energies up to $17 \mathrm{keV}$. Interestingly, a beam of $\mathrm{H}^{-}$ions at solar wind energies ( $1 \mathrm{keV}$ ) was detected by the electron spectrometer and was attributed to two stages of charge exchange [Burch et al., 2015]. The proton and pickup ion observations largely confirm the Rubin et al. [2014] predictions made using hybrid and multifluid simulations of the solar wind interaction with the distant comet 67P. In the current paper electron fluxes measured by the IES will be shown for the time period when the comet was near $3 \mathrm{AU}$.

The solar wind interaction with comets has been extensively studied over several decades with remote observations, in situ spacecraft data, and theoretically, although the emphasis has largely been on active comets in the inner solar system such as comet Halley in 1986 [cf. Galeev et al., 1985; Neugebauer et al., 1987; Coates et al., 1989; Johnstone et al., 1993; Cravens and Gombosi, 2004; Gombosi, 2015]. The driving process in the solar wind-comet interaction is the creation of cometary ions and their pickup and assimilation (or partial assimilation) into the solar wind flow. Cometary ions are created when neutrals (e.g., $\mathrm{H}_{2} \mathrm{O}, \mathrm{OH}, \mathrm{O}$, and $\mathrm{H}$ ) are ionized by solar radiation, by electron impact, or by charge exchange with solar wind protons. These are shown by the following representative equations, respectively:

$$
\begin{aligned}
& \mathrm{hv}+\mathrm{H}_{2} \mathrm{O} \rightarrow \mathrm{H}_{2} \mathrm{O}^{+}+e \\
& \mathrm{e}+\mathrm{H}_{2} \mathrm{O} \rightarrow \mathrm{e}+\mathrm{H}_{2} \mathrm{O}^{+}+\mathrm{e} \\
& \mathrm{H}^{+}+\mathrm{H}_{2} \mathrm{O} \rightarrow \mathrm{H}_{2} \mathrm{O}^{+}+\mathrm{H}(\text { fast })
\end{aligned}
$$

An electron produced in the first reaction (i.e., a photoelectron) has an energy equal to the photon energy (hv) minus the ionization potential of the neutral species (i.e., $\mathrm{H}_{2} \mathrm{O}$ or $\mathrm{CO}_{2}$, but we will only consider the former to make the calculations simpler). The charge exchange reaction does not produce an electron but does create a fast neutral $\mathrm{H}$ atom. The ionization potential depends on the photoion's final state. The newly created ions are accelerated by the solar wind motional electric field $\left(\mathbf{E} \approx-\mathbf{u}_{\mathrm{sw}} \times \mathbf{B} \approx 5 \times 10^{-4} \mathrm{~V} / \mathrm{m}\right.$ near $3 \mathrm{AU})$, where $\mathbf{u}_{\mathrm{sw}}$ is the solar wind velocity and $\mathbf{B}$ is the interplanetary magnetic field (IMF). The ions are also 
Table 1. Typical Solar Wind Parameters for a Heliocentric Distance of $3 \mathrm{AU}^{\mathrm{a}}$

\begin{tabular}{|c|c|c|c|c|c|c|}
\hline$n_{\text {sw }}\left(\mathrm{cm}^{-3}\right)$ & $u_{\mathrm{sw}}(\mathrm{km} / \mathrm{s})$ & $T_{\text {esw }}(\mathrm{K})$ & $B_{\mathrm{sW}}(\mathrm{nT})$ & $M_{\mathrm{ms}}$ & $r_{\text {psw }}(\mathrm{km})$ & $r_{\text {PUlsw }}(\mathrm{km})$ \\
\hline 0.5 & 500 & $10^{5}(\approx 10 \mathrm{eV})$ & 1 & 6 & $10^{3}$ & $2 \times 10^{4}$ \\
\hline
\end{tabular}

deflected by the magnetic field once they have been accelerated. Pickup ion trajectories start out being cycloidal with a length scale of several ion gyroradii $2 \pi r_{L}$ where the gyroradius is given by $r_{L} \approx \frac{u_{s w}}{\Omega} \approx 10^{4} \mathrm{~km}$. The gyrofrequency is $\mathbf{\Omega}=\frac{e \mathbf{B}}{m}$.

For an active comet, the pickup process is altered by wave-particle interactions associated with waves generated by the unstable cometary ion distribution function. Wave-particle interactions tend to pitch angle scatter ions and thus to further assimilate them into the solar wind flow [Johnstone et al., 1993]. The velocity vectors of initially created pickup ions have a discrete angle with respect to the magnetic field (or pitch angle). Magnetic perturbations tend to change the particle pitch angle but to leave the speed the same, and this is called pitch angle scattering [Li et al., 1997; Johnstone et al., 1993]. The mass loading of the plasma associated with the addition of heavy ions slows the flow down, and for an active comet a weak bow shock forms, as well as a stagnation region surrounding the nucleus [Galeev et al., 1985]. For comet Halley the radial distance of the bow shock was about 30 times greater than the heavy ion gyroradii $\left(r_{L} \approx 10^{4} \mathrm{~km}\right)$, but for weaker comets (e.g., comet Giacobini-Zinner) this ratio is smaller and the interaction is less "fluid like" [Koenders et al., 2013; Rubin et al., 2014].

The solar wind interaction with comet 67P at the end of 2014 when it was located near $3 \mathrm{AU}$ is very different than the solar wind interaction with active comets previously studied. The gas production rate was low $\left(Q \approx 10^{26} \mathrm{~s}^{-1}\right)$, and a typical IMF field strength at this heliocentric distance is low $(B \approx 1 \mathrm{nT})$. Furthermore, Rosetta was located very close to the nucleus $(r \approx 10-200 \mathrm{~km})$ [Gulkis et al., 2015; Nilsson et al., 2015; Goldstein et al., 2015]. Cometary ion gyroradii in the solar wind at $3 \mathrm{AU}$ are about $r_{g} \approx 3 \times 10^{4} \mathrm{~km}$, more than 1000 times greater than the radius of the nucleus. This means that the pickup ions right near the nucleus are "unmagnetized." Rubin et al. [2014] carried out multifluid and hybrid simulations for this type of interaction and made predictions for comet 67P. Also see Koenders et al. [2013, 2015]. Their results showed that cometary ions created near the nucleus are accelerated by the electric field and are initially slow moving such that the near-nucleus density $\left(n_{i} \approx 10-100 \mathrm{~cm}^{-3}\right)$ is much greater than the solar wind proton density $\left(n_{\mathrm{sw}} \approx 0.5 \mathrm{~cm}^{-3}\right)$. Table 1 summarizes solar wind conditions for $3 \mathrm{AU}$.

The current paper will follow up on the IES work shown by Goldstein et al. [2015], Burch et al. [2015], Clark et al. [2015], and Broiles et al. [2015] and will emphasize data interpretation using a test particle code plus an electron transport model. In particular, Clark et al. [2015] showed that electron fluxes near the comet were much higher than solar wind electron fluxes, and the energies $(\approx 100 \mathrm{eV})$ were much greater than solar wind electron energies $(\approx 10 \mathrm{eV})$. They suggested that dominant mechanisms are likely an admixture of photoelectrons and/or wave-particle interactions; however, they could not explicitly rule out other mechanisms such as shocks or compression enhancements. We will use a combination of simple models to try to explain these enhanced fluxes.

Our ion test particle model will confirm that the low-energy ions observed by IES are almost certainly cometary pickup ions $\left(\mathrm{H}_{2} \mathrm{O}^{+}\right.$or $\left.\mathrm{CO}_{2}{ }^{+}\right)$. The simulations also explain why a region of high ion (and electron) density (100 times greater than the solar wind density) surrounds the nucleus at least out to the distance of Rosetta (cometocentric distance, $r \approx 30-100 \mathrm{~km}$ ) and why this region is populated by slowly moving pickup ions (in agreement with the predictions of Rubin et al. [2014]). We will then explore the effects of electron sources including solar wind electrons, photoelectrons from ionization of coma gas, and photoionization of the surface of the nucleus. Not surprisingly, electrons, from either the coma gas or surface, must be partially confined to the vicinity of the nucleus by an ambipolar electric field as described by the generalized Ohm's law. In addition, our simple modeling will show that further energization of the electrons is needed, perhaps due to compression associated with a slowdown of the electron fluid. 


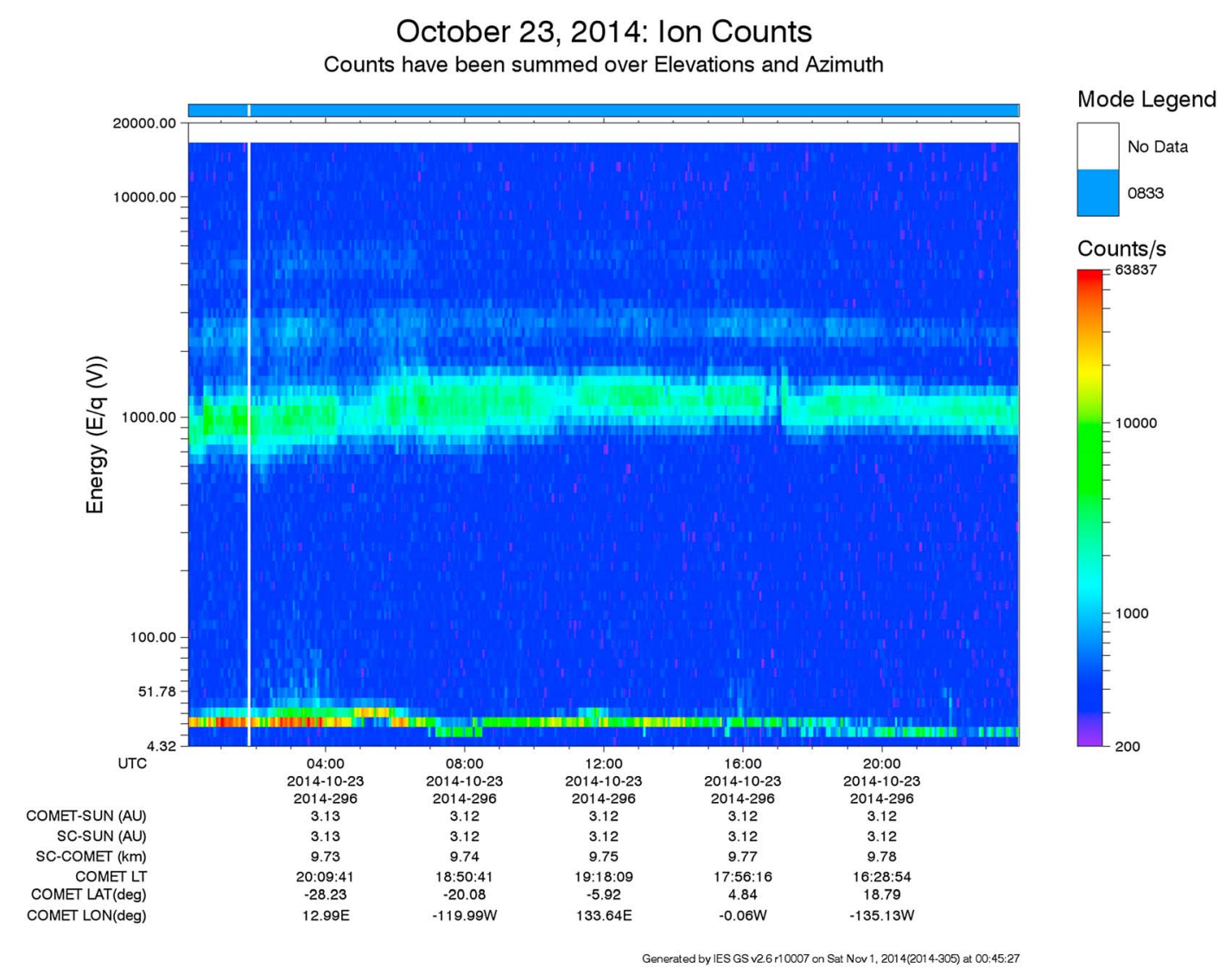

Figure 1. IES ion spectrogram for 23 October 2014. The color bar shows counts per second per energy bin for the ion sensor. The ordinate shows energy in units of $\mathrm{eV}$ and the abscissa is time (heliocentric and cometocentric distances are also shown). The higher-energy counts are due to solar wind protons and alpha particles, and the low-energy counts are thought to be cometary pickup ions.

\section{The Rosetta Ion and Electron Spectrometer (IES) and Data}

\subsection{The IES Instrument}

The IES component of the Rosetta Plasma Consortium (RPC) package was described by Burch et al. [2007]. It consists of two top-hat electrostatic spectrometers that measure electron and ion fluxes over a large energy range ( $5 \mathrm{eV} / \mathrm{e}$ up to $17 \mathrm{keV} / \mathrm{e})$ and over a large $\left(2.8 \pi \mathrm{sr}\right.$ ) field of view of $360^{\circ}$ in azimuth and $90^{\circ}$ in elevation. The angular resolution for both electron and ion measurements is $5^{\circ}$ in elevation, achieved by electrostatically sweeping 16 deflection voltages. There are 16 electron anodes, giving a resolution of $22.5^{\circ}$ in azimuth for electrons. For ions, nine anodes cover a $45^{\circ}$ part of the azimuth in the solar wind direction, giving a $5^{\circ}$ azimuth resolution for precise solar wind measurements, plus seven anodes that cover the rest of the azimuth, giving a $45^{\circ}$ resolution, in the nonsolar wind direction. About $25 \%$ of the field of view of IES is blocked by the spacecraft body as well as by other instruments mounted on the spacecraft. This blockage is mainly in low elevations of the field of view of IES. The current paper will primarily focus on IES electron data from the "central" anodes and elevation for which the instrument response is best known and spacecraft blockages are not an issue. Efforts are underway by the IES team to derive more accurately the moments of the electron distribution function.

IES counts are converted to physical fluxes by a conversion factor taking into account appropriate solid angles and detector efficiencies as discussed by Burch et al. [2007]. The count rate ( $\mathrm{d} C / \mathrm{d} t$ with units of $\mathrm{s}^{-1}$ ) for a given direction and energy is given in terms of the differential electron flux by the expression:

$$
\frac{\mathrm{d} C_{i j k}}{\mathrm{~d} t}=G_{i j}\left(E_{k}\right) E_{k} F\left(\mathbf{n}_{i j}, E_{k}\right)
$$




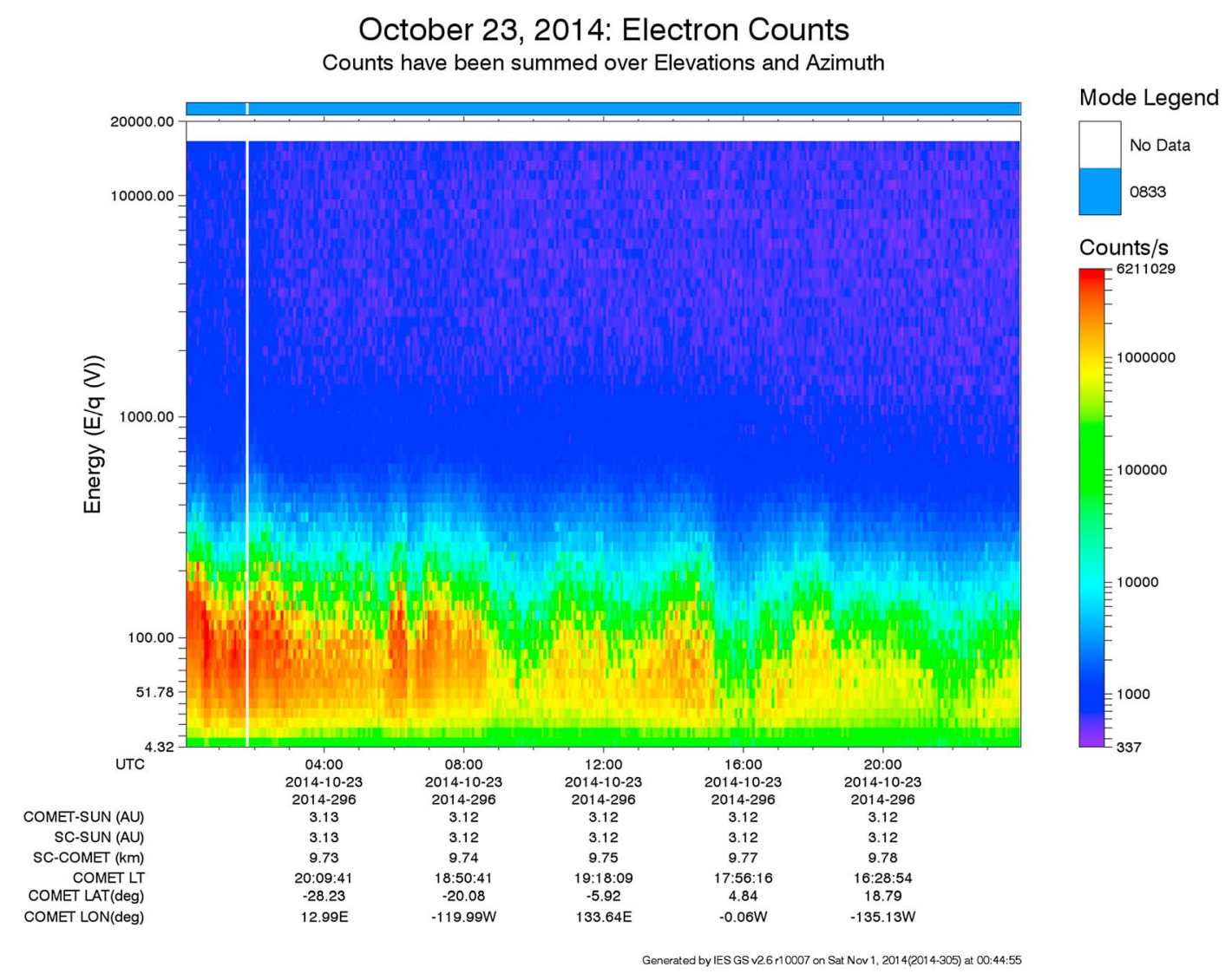

Figure 2. IES color spectrogram showing electron fluxes (color bar) versus energy (vertical axis) and time (horizontal axis). The time variations have been shown to be associated with the neutral density as measured by the Rosetta ROSINA instrument.

The instrument directional resolution was discussed above and the channels are denoted by the indices $i$ and $j$. The energies sampled by IES $\left(E_{k}\right)$ are labeled with index $k . F(n, E)$ is the differential flux (in $\mathrm{cm}^{-2} \mathrm{~s}^{-1} \mathrm{eV}^{-1} \mathrm{sr}^{-1}$ ) in the direction given by unit vector $\mathbf{n}$ and at energy $E$. The reported geometric factor, $G_{i j}\left(E_{k}\right)$, for IES is $6 \times 10^{-5} \mathrm{~cm}^{2}$ sr eV/eV for each ion pixel (per $5^{\circ}$ elevation angle, per $45^{\circ}$ azimuth angle) and $3 \times 10^{-5} \mathrm{~cm}^{2}$ sr eV/eV for electrons, but the geometric factor might change some in the future as calibration activities are continuing. Due to the nongyrotropic distribution expected for pickup ions, different IES pixels see different parts of the pickup ion distribution. Assuming a noise level of 2 counts per second per look direction per energy bin, the detectable differential energy flux threshold for IES would be $\sim 2 \times 10^{3} \mathrm{eV} /$ $\left(\mathrm{cm}^{2} \mathrm{~s} \mathrm{eV}\right)$. IES has 16 elevation bins and 16 anodes; however, due to data downlink constraints, for certain operation modes adjacent bins are first averaged and then transmitted.

\subsection{IES Data for Late 2014 and Early 2015-Ions}

Some examples of IES ion energy spectra are displayed in this section. The instrument started to detect low-energy ions in its lowest energy channels in mid-August of 2014, only a few weeks after the spacecraft's arrival at the comet. Figure 1 shows example spectrograms from the ion sensor of IES, for 23 October 2014, when the comet was at $3.12 \mathrm{AU}$ and the spacecraft was at a distance of $10 \mathrm{~km}$ from the comet. The lowenergy ions observed have been attributed to a negative spacecraft potential, attracting newly born ions to the instrument [Goldstein et al., 2015; Broiles et al., 2015]. As shown by Odelstad et al. [2015] using data from the RPC-LAP Langmuir probe instrument, electron fluxes were sufficiently high to start driving the spacecraft (s/c) to negative potentials already at first arrival at $30 \mathrm{~km}$ cometocentric distance in early September. On 23 October, the spacecraft potential was consistently negative at around -10 to $-20 \mathrm{~V}$. Higher-energy pickup ions have also been observed starting in September 2014 [Goldstein et al., 2015], and it is thought that these ions were energized by the solar wind motional electric field before reaching the spacecraft. 


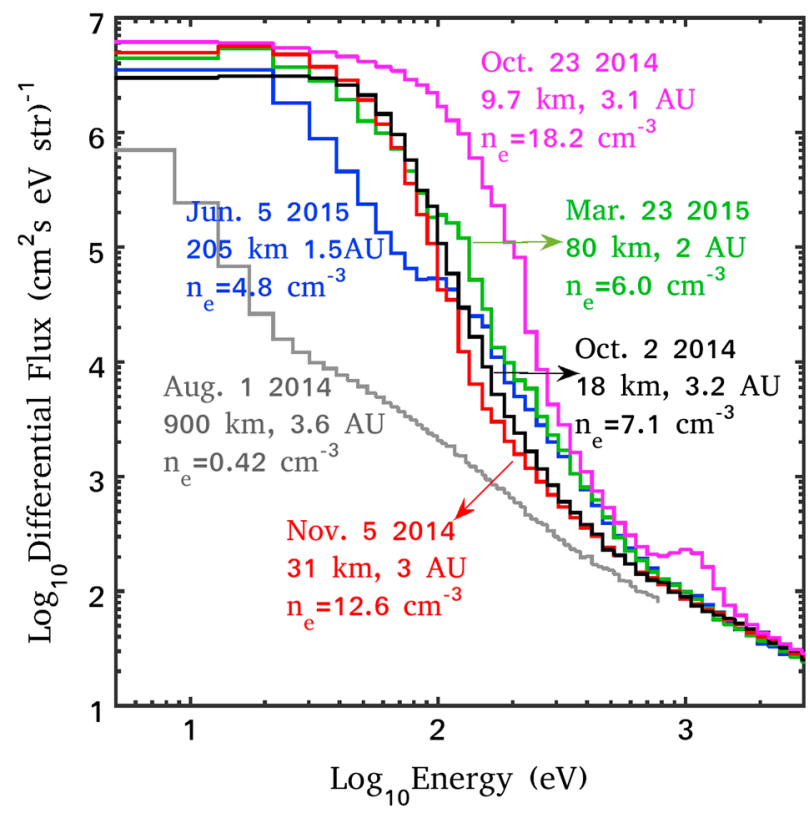

Figure 3. The daily averaged electron differential flux using IES measurements on 1 August 2014 (grey), 2 October 2014 (black), 23 October 2014 (purple), 5 November 2014 (red), 23 March 2015 (green), and 5 June 2015 (blue). The spectrum from 1 August 2014 shows a typical solar wind type electron flux. And the 23 October 2014 spectrum was measured during a CME event. Distance to the comet in kilometers and distance to the sun in AU for each spectrum are indicated on the figure. The other four spectra are typical differential fluxes at the given cometocentric distances. do the acceleration of pickup ions in the opposite direction, which is also seen in the data. These phenomena and measurements were discussed by Burch et al. [2015], Nilsson et al. [2015], Broiles et al. [2015], and Goldstein et al. [2015] and will not be emphasized in the current paper.

\subsection{IES Data for Late 2014 and Early 2015-Electron Fluxes}

A color spectrogram of IES electron fluxes is shown in Figure 2. The electron fluxes measured near the comet greatly exceed typical solar wind electron fluxes (section 7 will have more information on this), and the fluxes are large over a wide range of energies up to $100-200 \mathrm{eV}$.

Figure 3 shows the electron differential particle flux spectra for several days at different heliocentric and cometocentric distances. The spectra are daily averages of the IES measurements. The grey curve in Figure 3 shows a solar wind type spectrum from 1 August 2014, when Rosetta arrived in the vicinity of $67 \mathrm{P}$ at about $900 \mathrm{~km}$. The comet was at 3.6 AU from the Sun with a quite low gas production rate. This spectrum can be fitted with a bi-Maxwellian distribution with two suprathermal electron temperatures, hot and core (fit is not shown). Rosetta approached to closer distances to the comet as low as $9.5 \mathrm{~km}$ during October. The black curve in Figure 3 is from 2 October where the spacecraft is only $18 \mathrm{~km}$ away from the comet's center of mass and well within the coma. This spectrum is mainly composed of surface photoelectrons, photoelectrons from photoionization, and secondary electrons. On 22 October at around 16:30 UTC a solar wind pressure pulse (identified as a coronal mass ejection (CME), which had merged with a corotating interaction region (CIR) [Edberg et al., 2016]) hit the comet and lasted until midday of 23 October. For simplicity, we will refer to this event as the CME event of 23 October throughout the rest of this paper. The spectrum in purple is measured during this event. A feature in this spectrum at around $1000 \mathrm{eV}$ is particularly noticeable. By the end of October, the spacecraft started to move away from the comet while making measurements at constant orbits (e.g., $10 \mathrm{~km}$ and $30 \mathrm{~km}$ ) for several days. The red curve is from 5 November 2014, when Rosetta was $31 \mathrm{~km}$ from the comet. In February the spacecraft performed a series of maneuvers to make measurements at various distances (more discussion on this is given below 

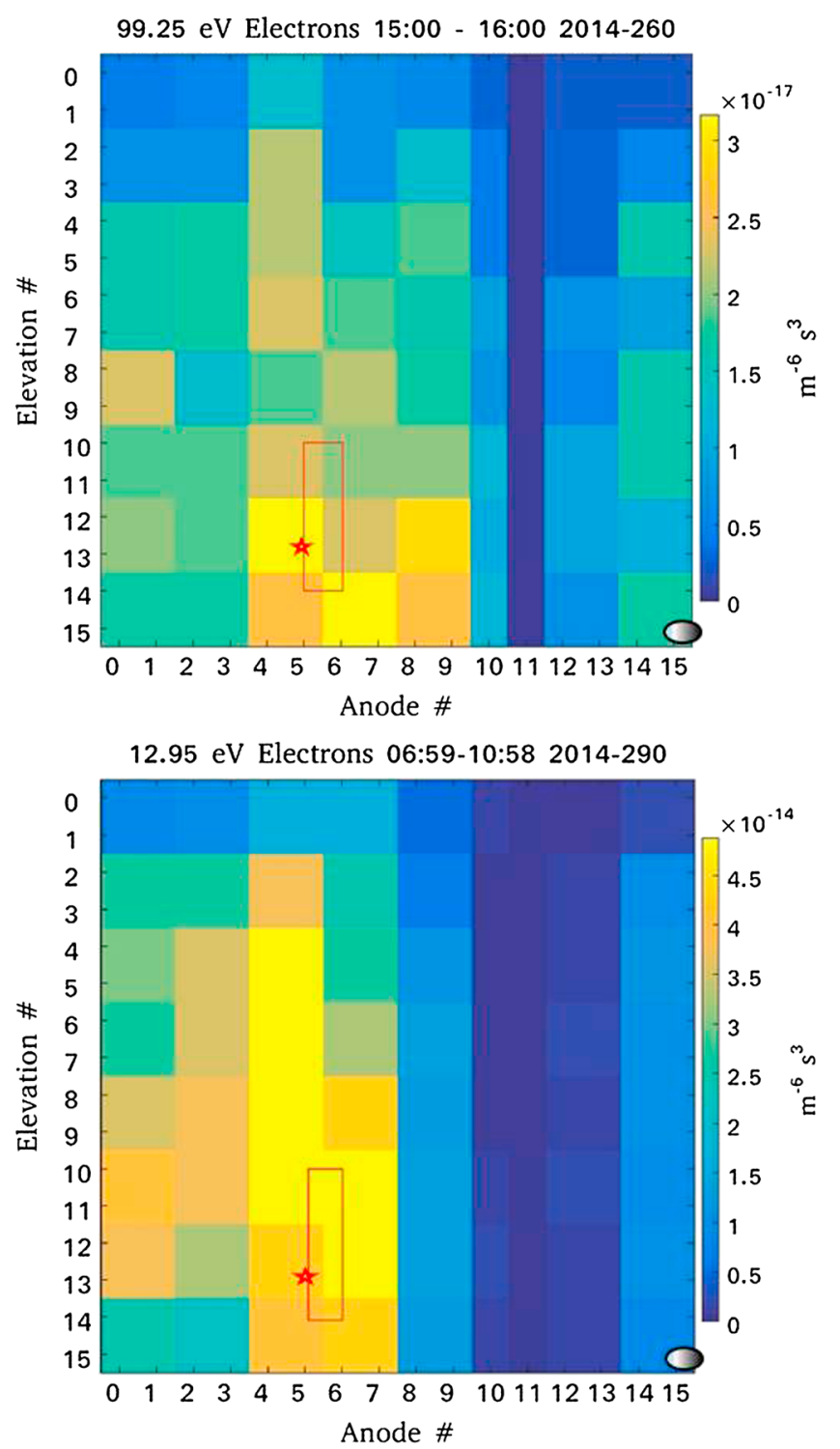

Figure 4. (top) Phase space distribution function for each of the IES panels using the corresponding count rates of $100 \mathrm{eV}$ electrons. The data is from 17 September 2014, averaged over 15:00-16:00 UTC. Color bar shows the amplitude of the phase space distribution function $\left(\mathrm{m}^{-6} \mathrm{~s}^{3}\right)$ in linear scale. The detection mode on this day combined every two adjacent elevation steps and anodes and energy steps. (bottom) PSD for 17 October 2014, 7:00-11:00 UTC for $13 \mathrm{eV}$ electrons. The IES mode is the same as the top plot. The color bar shows the amplitude of phase space distribution function $\left(\mathrm{m}^{-6} \mathrm{~s}^{3}\right)$. Electron counts are mostly seen near anodes 4-7 and elevation steps 10-14. Positions of the Sun and the comet in both plots are shown with a red star and a grey oval, respectively.
Figure 6). In late March 2015 the high-energy ion counts due to solar wind disappeared from the IES spectrograms indicating that a substantial amount of plasma is present near the comet to block the solar wind from reaching the spacecraft. The spectra in green and blue in Figure 3 are from 23 March and 5 June 2015 , when the comet is significantly more active.

The differential flux spectra are calculated from the instrument count rates according to equation (2). The IES detection panels record different electron count rates even when the spacecraft is inside a presumably isotropic plasma environment. There are several reasons for this, including blockage by other spacecraft instruments, IES detection efficiency, and spacecraft potential. The color bar in Figure 4 shows the phase space distribution function amplitude of electrons for individual panels of the IES electron sensor. Figure 4 (top) is for $100 \mathrm{eV}$ electrons on 17 September 2014, and Figure 4 (bottom) shows the $13 \mathrm{eV}$ electrons measured on 17 October 2014. The phase space distribution function for a given energy and direction is obtained by the following expression:

$$
f(E, \Omega, r)=\frac{m_{e}^{2}}{2 E} \frac{\mathrm{d} \varphi}{\mathrm{d} E d \Omega}
$$

where $\frac{d \varphi}{d E d \Omega}$ is the differential particle flux similar to the $F$ parameter in equation (2) and $m_{e}$ is the mass of the electron.

Each two adjacent elevation steps and anodes have been averaged, and counts are reported for the sum of the counts in two adjacent energy bins of the energy sweep. It is clear that anodes 4-7 and elevation steps 10-15 show a higher yield and are not subject to spacecraft blockage. Therefore, throughout this paper we only used the electron counts averaged over anodes 5-6 and elevations 10-14 and assumed an isotropic distribution to calculate the flux and density of electrons. It is possible in some measurements to observe a higher count in panels outside of the selected range, but we used the same set of panels for all measurements to expedite the data handling processes. 
Clark et al. [2015] examined electron spectra measured by IES over a several month time period and were able to fit the distribution function, $f(v)$, with the following expression:

$$
f(v)=A e^{-\left(\frac{v}{v_{0}}\right)^{p}}
$$

where $v$ is the electron speed, $A$ is an amplitude (i.e., distribution function for $v=0$ ), $v_{0}$ is related to average energy, and $p$ is a power exponent. Near $3 \mathrm{AU}$, Clark et al. found that $p \approx 5$ and $v_{0} \approx 3500 \mathrm{~km} / \mathrm{s}$.

\subsection{IES, LAP, and MIP Data-Densities and Average Energies}

We obtained suprathermal electron densities and their average energies for the measured spectra by assuming isotropy and simply by integrating such spectra using only the central anodes and elevations. In the future a more difficult moment analysis of the data will need to be undertaken (T. W. Broiles et al., Characterizing Cometary Electrons with Kappa Distributions, submitted to Journal of Geophysical Research, 2016). For isotropic plasma the electron density can be calculated by the following expression [cf. Khazanov, 2011; Cravens, 1997; Schunk and Nagy, 2009]:

$$
n_{e}=4 \pi \int_{0}^{\inf } f(v) v^{2} \mathrm{~d} v
$$

in the velocity space, and by

$$
n_{e}=2 \pi \sqrt{2 m_{e}} \int_{4.3 \mathrm{eV}}^{\inf } \frac{1}{\sqrt{E}}\left(\frac{\mathrm{d} \varphi}{\mathrm{d} E d \Omega}\right) \mathrm{d} E
$$

in the energy space.

Similarly, the average energy of electrons can be calculated using the following expression:

$$
\langle E\rangle=\frac{2 \pi \sqrt{2 m_{e}}}{n_{e}} \int_{4.3 \mathrm{eV}}^{\inf } \sqrt{E}\left(\frac{\mathrm{d} \varphi}{\mathrm{d} E d \Omega}\right) \mathrm{d} E
$$

The integration is done over the full range of IES energies starting from the first energy step. This means that the electrons with energies lower than a couple of eV (including any possible cold electron population) are not detected by the IES and will not contribute to our electron densities. Plus, a negative spacecraft potential will repel even more of the electron population.

Figure 5a shows the IES electron densities versus distance to the comet from early August 2014 to the end of February 2015. The data points are color coded based on the time of measurements. The data only include certain days where the IES has measured continuously in certain detection modes. Figure $5 \mathrm{~b}$ shows the density time series when Rosetta hovered around the comet at about $10 \mathrm{~km}$ for a couple of days in October. The IES electron densities (blue dots) are compared with the LAP (green dots) ion densities [Edberg et al., 2015] and the MIP-LDL and SDL (grey and black dots, respectively) electron densities for the time period between 16 October to 28 October 2014. The RPC-MIP experiment is a mutual impedance probe which retrieves electron densities from the estimated position of the plasma frequency in the MIP complex (amplitude and phase) mutual impedance spectra [Trotignon et al., 2007, and references therein]. Data from both the socalled short Debye length (SDL) and long Debye length (LDL) modes of the MIP experiment are used. Note that the LDL mode enables us to estimate the electron density in the range $30-350 \mathrm{~cm}^{-3}$, implying that MIP is blind to plasma densities below and above this observational window when operated in this mode. On the other hand, the SDL mode allows measurements of only very large densities.

Also shown, in Figure $5 \mathrm{c}$ on the right axis, is the average energy of suprathermal electrons calculated from the IES data. Typical $E$ is around $10-30 \mathrm{eV}$ but reached $80 \mathrm{eV}$ on 23 October. The time of the CME event is also marked with a vertical dashed line in Figures $5 b$ and $5 c$. The CME event on 23 October 2014 triggered a very negative spacecraft potential such that LAP electron densities could not be determined on that day and MIP electron densities and LAP ion densities were used for comparison.

The derived IES densities are $\approx 3-20 \mathrm{~cm}^{-3}$ (Figure $5 \mathrm{a}$ ), where typical solar wind densities at $3 \mathrm{AU}$ are $\approx 0.5 \mathrm{~cm}^{-3}$. Large time variations are evident with excursions up to about $100 \mathrm{~cm}^{-3}$ and appear to be linked with neutral density variations as measured by the ROSINA COPS sensor onboard Rosetta [Edberg et al., 2015]. 


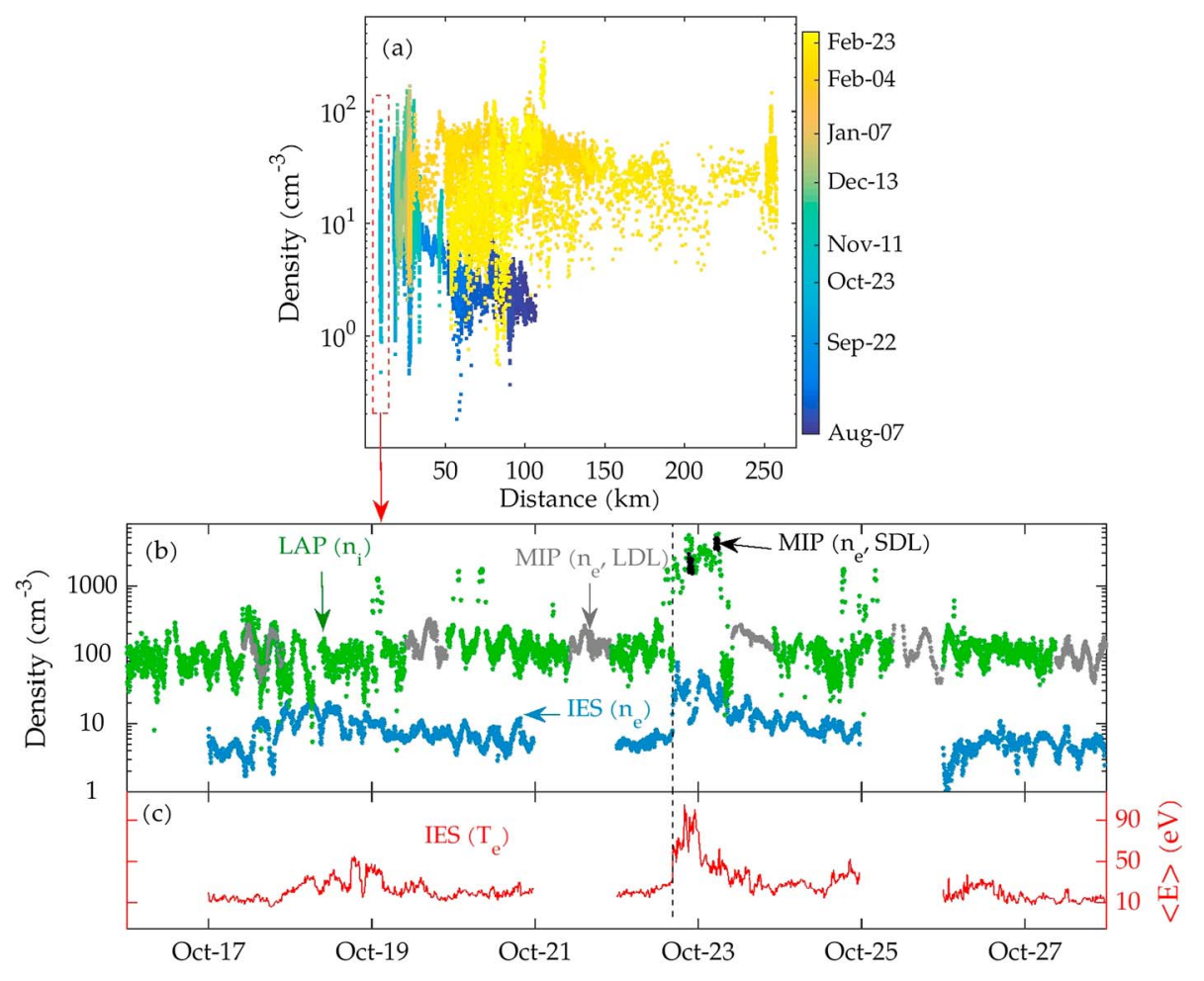

Figure 5. (a) Electron density versus distance to the comet. The data includes all days between August 2014 to February 2015 for which IES operated continuously in certain modes. The points are color coded based on the time of measurements. The time series for the points near $10 \mathrm{~km}$ (indicated by the red rectangle) is shown in Figures $5 \mathrm{~b}$ and $5 c$, which correspond to the data between 16 October to 28 October. (b) The IES electron densities (blue dots), LAP ion densities (green dots), and MIP electron densities (grey dots for LDL mode and black dots for SDL mode) are shown. (c) The average suprathermal electron energy from IES data is shown with the red curve with the corresponding values on the right axis. The vertical dashed line near 23 October shows the onset of the enhanced count rate observations due to the CME event.

Also see Figure 1 in Goldstein et al. [2015]. The electron and ion densities measured by LAP and MIP [Edberg et al., 2015] are very comparable and in principle include electrons of all energies. These densities are several times greater than the IES densities that are suprathermal electron densities, due to different detection methods. Evidently, a population of electrons with energies less than $\approx 5 \mathrm{eV}$ exists that IES cannot detect, especially if there is a high spacecraft potential. There seems to be an anticorrelation in density variations between the LAP/MIP and the IES. A dip in the LAP (MIP) ion (electron) densities coincides with an enhancement in the IES data. Given the uncertainties in our methodology, there are a couple of possibilities to explain this anticorrelation. It can be a direct result of the bulk plasma flow, or it can be due to the change in the direction of the incoming flow into the IES caused by the spacecraft potential and pointing. Given the correlation between ROSINA/COPS neutral density measurements and LAP (MIP) ion (electron) densities [Edberg et al., 2015], it can also be argued that the anticorrelation between IES and LAP (MIP) ion (electron) densities stems from the fact that at each upsurge of neutral species (i.e., $\mathrm{H}_{2} \mathrm{O}, \mathrm{CO}_{2}$, and $\mathrm{CO}$ ), the suprathermal electrons become thermalized through electron neutral collisions. In addition, secondary and tertiary electrons are produced, which will contribute to the LAP/MIP measured densities. The suprathermal electron distribution can have solar wind origin or can be produced by photoionization, or it can be accelerated inward through various processes such as ambipolar electric field.

Questions suggested by these IES observations include (1) what causes the near-nucleus density enhancement? and (2) what explains the shape (and high energies) of the electron energy spectra?

The Langmuir probe (RPC-LAP) on Rosetta indicated that the spacecraft $(\mathrm{s} / \mathrm{c})$ had an electrical potential relative to the ambient plasma of about $V_{\mathrm{s} / \mathrm{c}} \approx-5 \mathrm{~V}$ to $-20 \mathrm{~V}$ [Edberg et al., 2015; Odelstad et al., 2015]. It is well-known that spacecraft, dust grains, etc., can acquire electrical charge (and thus potential) in space due to several types of charging currents [Hsu et al., 2012], including ambient plasma electron and ion 


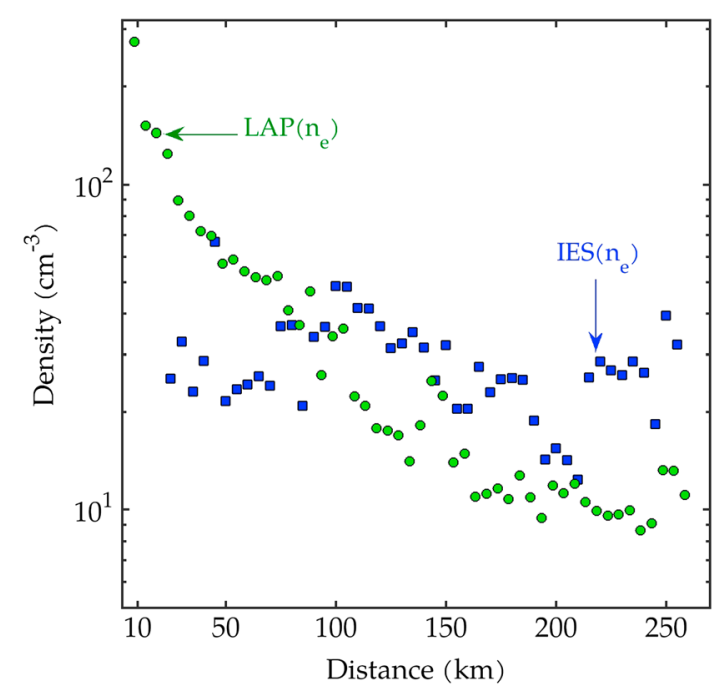

Figure 6. Electron density versus distance to the comet. The data are from 4-28 February 2015. The IES electron densities averaged every $5 \mathrm{~km}$ are shown with blue squares, and the LAP $5 \mathrm{~km}$ averaged electron densities [Edberg et al., 2015] are shown with green circles. The IES data from 16 February when Rosetta descended to distances as close as $8.5 \mathrm{~km}$ is not included. currents and photocurrents from absorption of solar photons. The cometary nucleus itself at $3 \mathrm{AU}$ should have an electrical potential. Furthermore, the spacecraft potential affects the comparison of model spectra with IES data, in which lower energy (less than the potential) electrons cannot be detected by the instrument.

In February 2015, a series of maneuvers by Rosetta provided an opportunity to compare the IES electron densities with other instruments at low and high distances.

In Figure 6, the IES electron densities averaged every $5 \mathrm{~km}$ are shown with blue squares and LAP electron densities (5 km average) [Edberg et al., 2015] are presented with green circles. The LAP data extend to smaller distances than IES as the close approach occurred on 16 February; this day is not included in the analysis due to bad quality of the IES data. The IES electron densities are higher than the LAP measured densities when Rosetta is far from the comet $(r>100 \mathrm{~km})$, and this relation tends to reverse as the spacecraft moves closer to the comet.

LAP measures all electrons where IES only measures suprathermal electrons, so one would expect the LAP densities to always exceed the IES densities. However, at far distances where densities are low, the spacecraft potential is positive and both LAP and IES data will in these circumstances also be perturbed by the cloud of spacecraft photoelectrons, particularly for LAP, which measures the integrated flux from all populations. However, the LAP electron density estimates assume the electron temperature can be correctly deduced from the LAP sweeps. Also note that the IES densities are only calculated approximately using just part of the distribution function. A reasonable interpretation of Figure 6 is that beyond $r \approx 100 \mathrm{~km}$, the overall electron population is hot and IES observes most of the electron distribution, whereas close to the nucleus a cold $(E<4 \mathrm{eV})$ electron population is present and is not observed by IES.

\section{The Neutral Coma of Comet 67P}

In the next few sections we use simple models to interpret IES data and such models start with the neutral density. Visual pictures of the comet taken by cameras onboard Rosetta reveal active regions on the comet that create an inhomogeneous environment of dust and neutral species around the comet. Neutral pressure measurements by the ROSINA/COPS instrument [Balsiger et al., 2007] also observed this anisotropy attributed to the complicated shape and illumination pattern on the nucleus combined with the comet's rotation [Bieler et al., 2015]. Previous attempts have been made to simulate the effect of asymmetric neutral gas distributions and jets in the plasma environment of a comet [e.g., Jia et al., 2008; Wiehle et al., 2011], and it has been established that the presence of neutral anisotropy around the comet affects the interaction of the solar wind with cometary plasma.

For the sake of simplicity we hereafter assume spherical symmetry for the neutral density at a cometocentric distance $r$ :

$$
n_{n}(r)=\frac{Q}{4 \pi u_{n} r^{2}}
$$

where $Q \approx 10^{26} \mathrm{~s}^{-1}$ is the gas production rate of the comet at $3 \mathrm{AU}, u_{n} \approx 1 \mathrm{~km} / \mathrm{s}$ is the neutral outflow speed, and $r$ is the cometocentric distance. A Haser model [Haser, 1957] was used to obtain the densities of daughter 


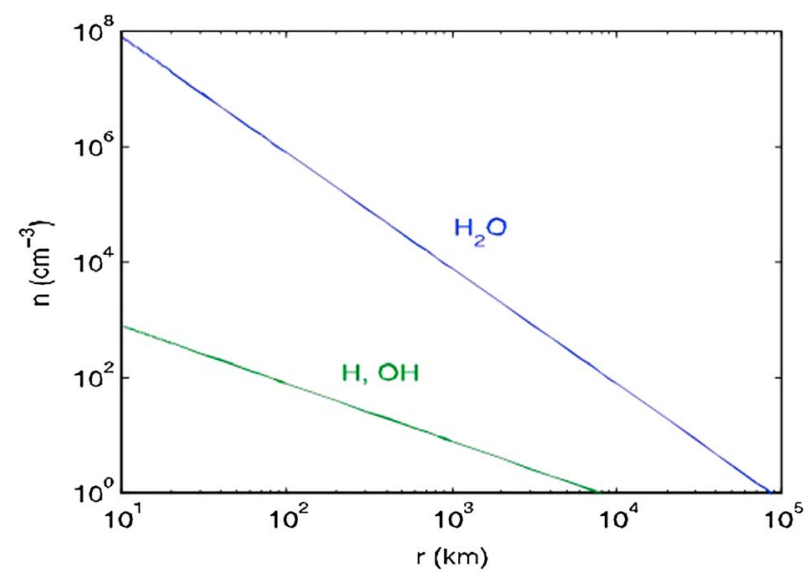

Figure 7. Neutral density profiles of cometary coma neutrals at $3 \mathrm{AU}$ are shown for a gas production rate of $10^{26} \mathrm{~s}^{-1}$ and an outflow velocity of $1 \mathrm{~km} / \mathrm{s}$. Parent $\left(\mathrm{H}_{2} \mathrm{O}\right)$ and daughter $(\mathrm{H}, \mathrm{OH})$ densities are constructed using a Haser model of the coma.

near the surface of the comet at $3 \mathrm{AU}$. Considerable variability in the neutral coma was seen by ROSINA associated with the rotation of the comet [Hassig et al., 2015]. Nonetheless, on average, our neutral profile is in agreement with ROSINA observations.

\section{Description of the Models}

\subsection{Test Particle Model and Results for lons}

We constructed a test particle model to study the energy and spatial distribution of cometary ions near the nucleus. The model is essentially the same as one used by Rahmati et al. [2014, 2015] for Mars and details can be found there. $\mathrm{H}_{2} \mathrm{O}^{+}$ions are created at rest in the comet rest frame with a production rate of $P(r)=\ln n(r)$, where $I \approx 10^{-7} \mathrm{~s}^{-1}$ is the ionization frequency appropriate for a heliocentric distance of $3 \mathrm{AU}$. The model includes both photoionization of neutrals by solar radiation [cf. Cravens et al., 1987] and charge exchange of solar wind protons. We will show later that electron impact ionization can also make a comparable contribution to the ionization but do not include this. Note that for our current purposes, the type of neutral species is not important as long as it is heavy (i.e., $m=18 \mathrm{amu}$ is used but some $\mathrm{CO}_{2}{ }^{+}$should also be present with $m=44 \mathrm{amu}$ ). New ions were created randomly with probabilities proportional to the neutral density.

Ion trajectories were found by analytically solving the equation of motion $\left(\boldsymbol{F}=m \frac{\mathrm{d} \boldsymbol{v}}{\mathrm{dt}}\right)$ with the Lorentz force, $\mathbf{F}=e(\mathbf{E}+\mathbf{v} \times \mathbf{B})$, where $\mathbf{v}$ is the particle velocity at any given time, $\mathbf{E}$ is the electric field, and $\mathbf{B}$ is the magnetic field (e.g., the interplanetary magnetic field). By taking advantage of the analytical solutions to the equations of motion of pickup ions, a fast method to calculate the cometary ion density and energy spectra was constructed [Rahmati et al., 2015], which is more efficient than traditional test particle models. An ion mass of $m=18$ amu was adopted. The solar wind motional electric field, $\mathbf{E} \approx-\mathbf{u}_{\mathrm{sw}} \times \mathbf{B}$, was adopted, where $\mathbf{u}_{\mathrm{sw}}$ is the solar wind velocity. The solar wind speed used was $400 \mathrm{~km} / \mathrm{s}$ and a magnetic field strength of $B \approx 2 \mathrm{nT}$ was adopted in order to account for some increase of the magnetic field right near the nucleus [Rubin et al., 2014]. The ambipolar electric field, however, was not included in this model as it caused instability in the current model.

Figure 8 (left) shows the differential flux of pickup ions at $10 \mathrm{~km}$ upstream of the comet and includes comparisons with IES data. Shown in Figure 8 (right) are results calculated for five different angles between the IMF direction and the solar wind flow direction. It is seen that a perpendicular angle gives higher-energy pickup ions but lower densities close to the comet. An enhancement in the ion density of about 1 order of magnitude is predicted for small angles between the solar wind flow direction and the IMF. The high-energy (10 keV or more) ion flux is low because such ions are born upstream of the nucleus by about a gyroradius $\left(10^{4} \mathrm{~km}\right)$ where the neutral density (and ion production rate) is low. lons with energies less than $\approx 100 \mathrm{eV}$ have high fluxes because they are created in the higher neutral density coma where the ion production rate is high. 

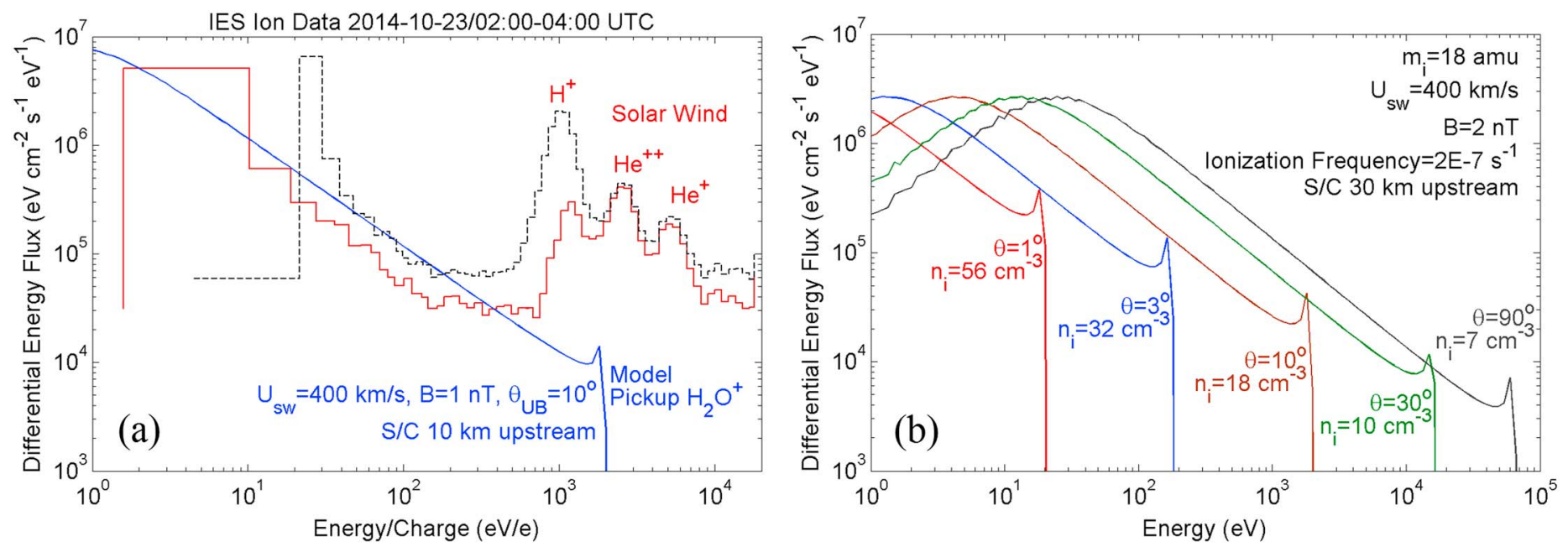

Figure 8. (left) Cometary ion differential energy flux from the test particle model versus ion energy for $\Theta_{\mathrm{UB}}$ (solar wind-IMF angle) $=10^{\circ}$ and IES data for 23 October 2014/02:00-04:00 UTC. Differential energy flux of $\mathrm{H}_{2} \mathrm{O}^{+}$ions calculated by the test particle model for a location $10 \mathrm{~km}$ upstream of the comet are shown. A solar wind velocity of $400 \mathrm{~km} / \mathrm{s}$ is used in the model, giving a maximum pickup $\mathrm{H}_{2} \mathrm{O}^{+}$energy of $2 \mathrm{keV}$. The black dashed line is raw IES data for the sunward anodes and including all elevations. The red curve is corrected for $-20 \mathrm{~V}$ spacecraft potential and includes only elevations with significant pickup $\mathrm{H}_{2} \mathrm{O}^{+}$flux. Note that most of the solar wind protons are deflected and therefore not showing up in the elevations included in the red curve. The blue curve is model results for conditions shown in the figure. (right) Cometary ion differential energy flux spectra from the test particle model, plus total ion densities for several values of $\Theta_{\mathrm{vB}}$ are shown. Differential flux of $\mathrm{H}_{2} \mathrm{O}^{+}$pickup ions at $30 \mathrm{~km}$ upstream of the comet, calculated for five different angles between the IMF direction and the solar wind flow direction. A 1 order of magnitude enhancement in ion density is predicted for small angles.

Note that the solar wind proton flux for the coronal mass ejection/interplanetary shock event of 23 October 2014 (shown in Figure 8 (left)) is about 10 times greater than that of a typical solar wind flux. If we include this in our ion models, the $\mathrm{H}_{2} \mathrm{O}^{+}$production frequency would be $\approx 5 \times 10^{-7} \mathrm{~s}^{-1}$ rather than $10^{-7} \mathrm{~s}^{-1}$ and would increase the model pickup ion fluxes by this factor, making them somewhat higher than the IES ion fluxes.

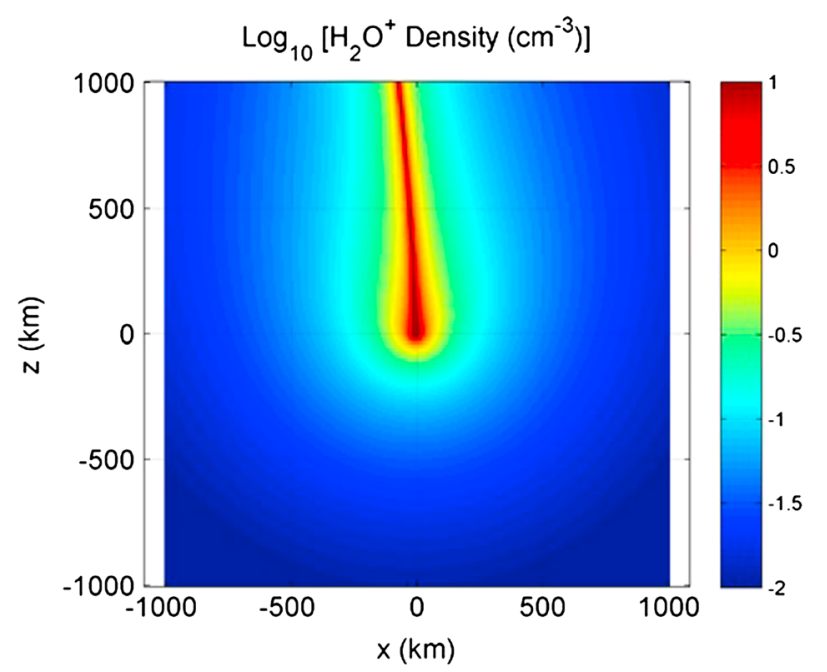

Figure 9. Cometary ion density contours from a test particle model of pickup $\mathrm{H}_{2} \mathrm{O}^{+}$ions near the nucleus of comet 67P. The density elongation is in the direction of the motional electric field and the Sun is to the right. The orientation at any given time depends on the interplanetary magnetic field direction. lon densities are shown in the color bar (log-10 of the density). Peak densities right near the nucleus are about $100 \mathrm{~cm}^{-3}$ and the overall structure is about $100 \mathrm{~km}$ in extent.
$\mathrm{CO}^{+}, \mathrm{O}_{2}{ }^{+}$, and $\mathrm{CO}_{2}{ }^{+}$ions from the ionization of the respective neutral species would experience a very similar process to the pickup $\mathrm{H}_{2} \mathrm{O}^{+}$ions shown in the figure but the curves would extend to higher energies by the ratio of the species mass to the $\mathrm{H}_{2} \mathrm{O}$ mass. However, the fluxes of these other ion species would be only a few percent of the $\mathrm{H}_{2} \mathrm{O}^{+}$fluxes shown in the figure, because the neutral densities are lower than the water density.

The spatial distribution of ion density from the model was also determined (Figure 9). For the model, the $x$ axis is pointing toward the Sun and the $z$ axis is perpendicular to the ecliptic plane. The magnetic field has a positive $y$ component, resulting in a motional electric field in the positive $z$ direction. A plume-type structure is evident in the pickup ion density in the figure, and it extends in the direction of the motional electric field. The 


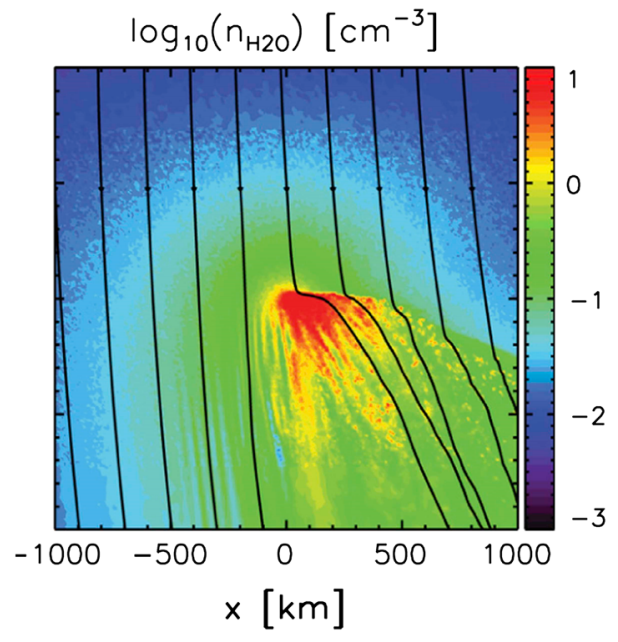

Figure 10. Figure 2 from the Rubin et al. [2014] hybrid model predictions of the solar wind interaction with the comet. A color bar is shown for the plasma density. The solid lines indicate the electric field direction. The enhanced density region is evident. Also note the antisolar deflection of the convection electric field near the nucleus. The Sun is to the left. model results extend further than shown in Figure 9, and at very large distances the plume curves to the left as expected for the cycloid trajectories of pickup ions born near the comet. This zeroth order approximation is somewhat different from the predictions of Rubin et al. [2014] close to the nucleus. As seen in Figure 2 of Rubin et al. [2014] (replicated in our Figure 10), or in a similar figure by Vigren et al. [2015], the pickup ion density distribution near the nucleus is more spread out and contains more structure, possibly due to the ambipolar electric field and/or due to wave-particle interactions captured by the hybrid or multifluid codes. Both Rubin et al. [2014] and our simulation show that for distances less than a few hundred kilometers from the comet, pickup ion densities are about an order of magnitude higher than unperturbed solar wind proton densities. As we will discuss later, the electric field near the nucleus is not just the motional electric field but should include the polarization (or ambipolar) electric field, which tends to confine electrons and expel ions in order to maintain quasi-neutrality. This could help to explain the differences between our simple model and the Rubin et al. [2014] study.

\subsection{Two-Stream Electron Transport Model}

Sources of electrons near the comet include (1) solar wind electrons, (2) photoelectrons from the ionization of cometary neutral gas, and (3) photoelectrons from the ionization of the surface of the nucleus. Solar photons with energies exceeding the ionization potential create ions and photoelectrons via photoionization of neutrals. We used the same solar extreme ultraviolet irradiance model as Richard et al. [2015] and Madanian et al. (Solar cycle variations in ion composition in the dayside ionosphere of Titan, submitted to Journal of Geophysical Research, 2016) for solar minimum conditions but adjusted for the comet's heliocentric distance. We used photoionization and photoabsorption cross sections for water, $\mathrm{CO}_{2}$ and $\mathrm{CO}$ [Gan and Cravens, 1990; Hansen et al., 2007], and we used the neutral densities shown in Figure 7 to calculate the photoelectron production rate as a function of cometocentric distance and electron energy. The electron production rates were then used as inputs to a two-stream electron transport code that determines steady state electron fluxes as a function of position and energy [cf. Gan and Cravens, 1990]. An appropriate set of electron impact cross sections was used in this code. We adopted magnetic field lines that are draped around the nucleus starting near the nucleus $(r \approx 10 \mathrm{~km})$ and extending into the solar wind at large distances [Gan and Cravens, 1990; Hansen et al., 2007]; see Figure 11a. We found that the details of the magnetic topology are not important unless we are dealing with conditions inside a diamagnetic cavity. For the time periods considered in this paper a cometary diamagnetic cavity has not formed yet and is the focus of our future studies. We adopted zero input electron flux at the outer boundary for a case with no solar wind, and for other cases we assumed that the incoming electron flux was the solar wind electron flux. And for yet other cases we imposed an electrostatic potential at the boundaries designed to approximately simulate the effects of a parallel ambipolar electric field (discussed in section 5). Some predicted suprathermal electron fluxes from these codes were shown by Hansen et al. [2007] for a more active comet 67P appropriate for perihelion.

We also carried out other calculations that neglected the coma gas but included photoelectrons produced directly from the surface of the nucleus by photoionization by solar radiation. This would only be applicable for locations where magnetic field lines connect the nucleus to the spacecraft. For this case, we adopted a $5 \%$ photoelectron emission efficiency at all photon wavelength/energies [Weingartner and Draine, 2001] and a $5 \mathrm{eV}$ surface work potential. 

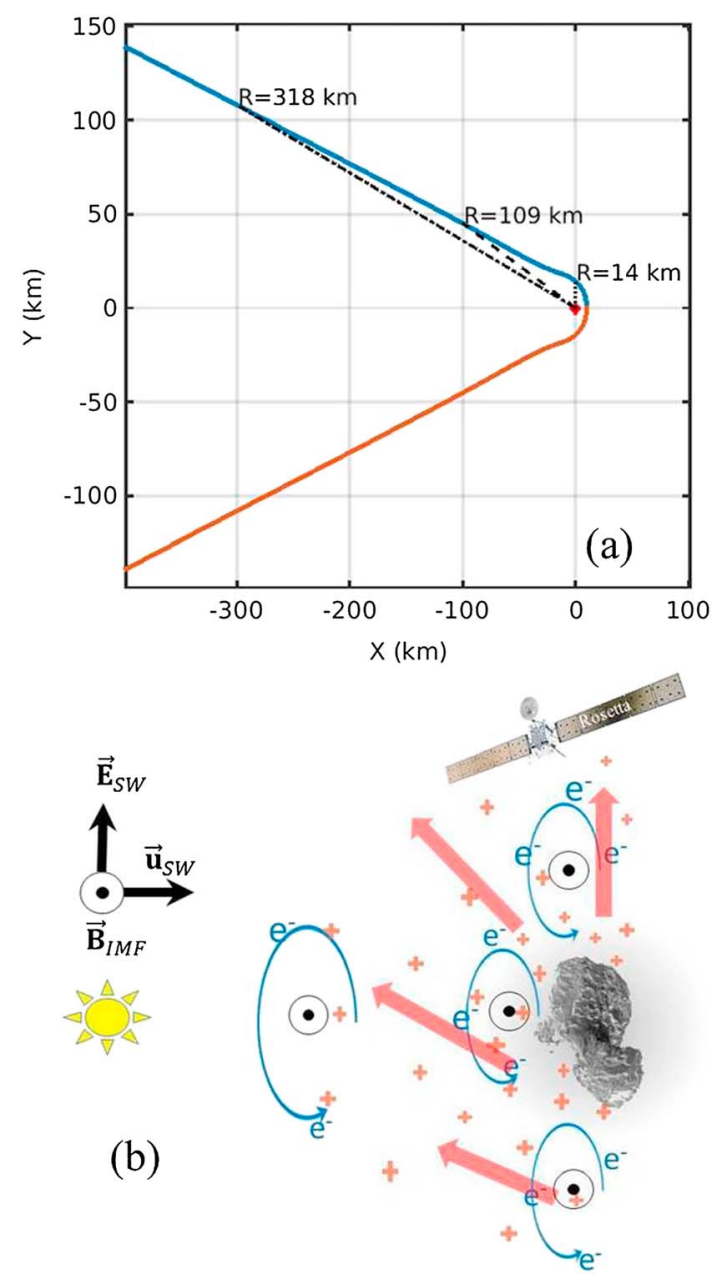

Figure 11. (a) Magnetic field geometry adopted for the two-stream electron model. The full extent of a parabola is shown with the symmetry axis around $Y=0$ line. The model only uses a half parabola (either the blue segment or the red). The closest the field line approaches to the nucleus is $10 \mathrm{~km}$ at the apex. Radial distances at some other locations are indicated. The nucleus is located at the origin and the Sun is to the right. (b) Schematic of plasma and fields within $100 \mathrm{~km}$ of the nucleus of the comet. Solar wind electrons and photoelectrons are indicated as well as cometary pickup ions. The location of the spacecraft is about $10 \mathrm{~km}$ from the nucleus. The electric field, which is a combination of motional electric field and ambipolar field, is also shown schematically as red arrows. The magnetic field is assumed to be out of the page. pressure is the second moment of the electron distribution function and is the average kinetic energy per unit volume in the electron bulk flow frame of reference $\left(p_{e} \approx n_{e}\langle\mathrm{KE}\rangle\right)$. The overall electrical potential difference along a magnetic field line between the center of the ion density structure and the outside is roughly the average electron kinetic energy divided by the electron charge: $\Delta V_{\mathrm{pol}} \approx \frac{\langle\mathrm{KE}\rangle}{e}$. For comet 67P near $3 \mathrm{AU}, \Delta V_{\mathrm{pol}} \approx 10-100 \mathrm{~V}$ judging by the fact that typical electron energies in Figure 3 are $10-100 \mathrm{eV}$. Electrons created within the dense plasma region with energies less than $\sim \Delta V_{\text {pol }}$ will be confined (or partially confined) to the vicinity of the nucleus. However, other effects such as $\mathbf{E} \times \mathbf{B}$ drift can remove electrons from this region. External solar wind electrons are accelerated inward (roughly toward the nucleus) by this potential structure and have their energies enhanced by $\sim e \Delta V_{\text {pol }}$. The motional electric field $\left(\mathbf{E}=\mathbf{u}_{e} \times \mathbf{B}\right)$ contribution to $\mathbf{E}$ from the $\mathrm{GOL}$ is also undoubtedly altered from its unperturbed solar wind value by the interaction with the comet. 


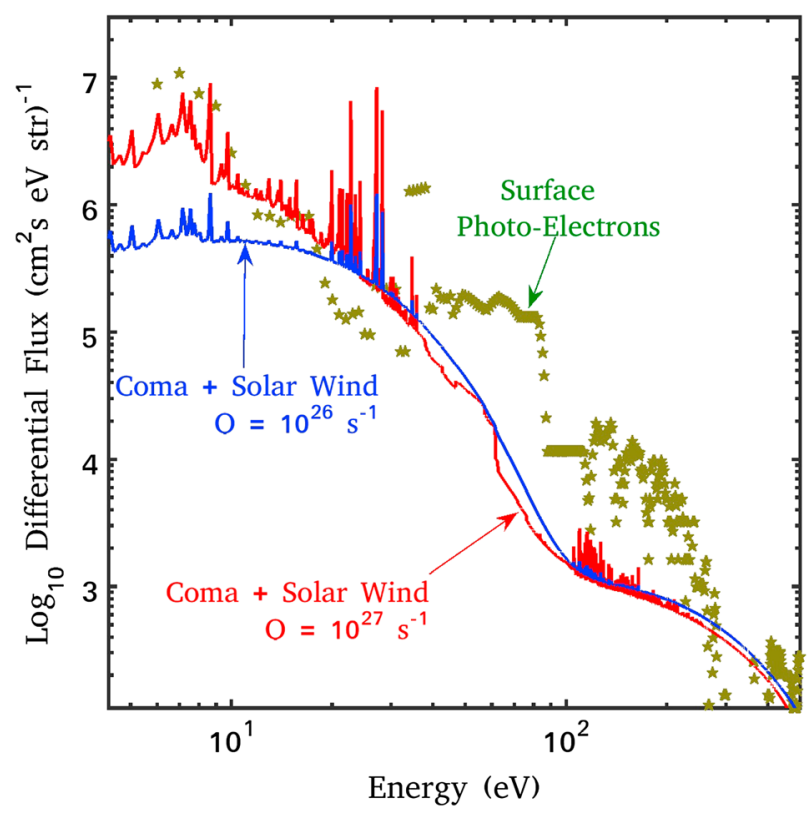

Figure 12. Model spectra for coma photoelectrons plus solar wind electrons at $10 \mathrm{~km}$ from the two-stream code for two cases: cometary gas production rate of $10^{26} \mathrm{~s}^{-1}$ and $10^{27} \mathrm{~s}^{-1}$ (possible outburst). The nucleus outgassing production rates for the two cases are indicated in the units of $s^{-1}$. A model spectrum for photoelectrons from the surface of the nucleus is also shown as the stars (one for each bin in the solar spectrum model). The photoelectric efficiency at the nucleus is assumed to be $5 \%$ for the latter.

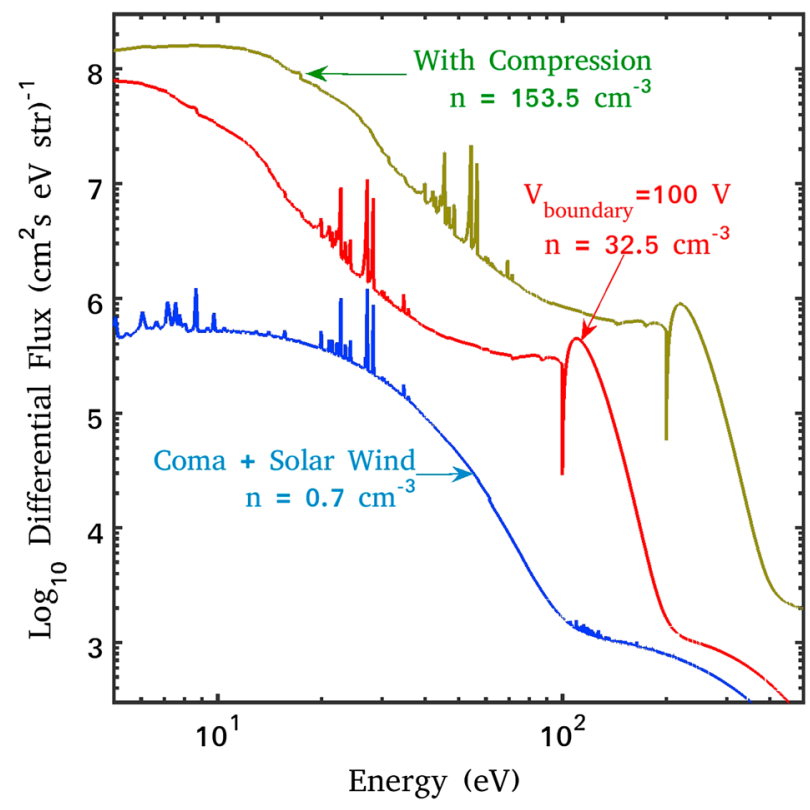

Figure 13. Model coma electron and solar wind electron fluxes at $10 \mathrm{~km}$ from the nucleus with ambipolar electric field and/or compression included. The blue curve is the model with no ambipolar potential and no compression. The red curve model includes the ambipolar field (along the magnetic field), and the green curve also puts in some compression $(K=2)$ as described in the text. The densities shown in the figure are calculated for energies greater than $5 \mathrm{eV}$. The density of the blue curve for all energies is $1.1 \mathrm{~cm}^{-3}$.
Figure $11 \mathrm{~b}$ illustrates our idea of what the plasma environment near the nucleus might look like. Near $3 \mathrm{AU}$, pickup ions initially move in the direction of the convection electric field and are not affected by the magnetic field near the nucleus [see also Rubin et al., 2014]. The ambipolar field is indicated in the schematic, and this should partially confine lower energy photoelectrons to the ion density structure. Solar wind electrons will be accelerated toward the density enhancement region by this electric field.

\section{Model Cases for Cometary Suprathermal Electrons and Results}

The ion density structure (Figure 9) is associated with an ambipolar electric field that confines the photoelectrons created within the ion density structure. This tends to boost the overall electron density so that it equals the ion density so as to maintain quasi-neutrality. Modeling the electron distribution function near the comet is very difficult. We considered several model cases: (1) an electron flux obtained when solar radiation directly photoionizes the surface of the nucleus sending electrons directly along the magnetic field to the spacecraft; (2) Photoelectrons from photoionization of the coma gas plus solar wind electrons $\left(0.7 \mathrm{~cm}^{-3}\right.$ density at $10 \mathrm{eV}$ temperature and $0.005 \mathrm{~cm}^{-3}$ at $100 \mathrm{eV}$ ) and modeling these with the two-stream model for draped magnetic field but without an ambipolar field; (3) spectra from the two-stream code with coma photoelectrons plus solar wind electrons but including a modified boundary condition designed to approximate the effects of $E_{\text {pol; }}$ and (4) the model electron fluxes from the previous case but also imposing the effects of a compression of a fluid parcel near the nucleus such as might take place at an electrostatic shock. 


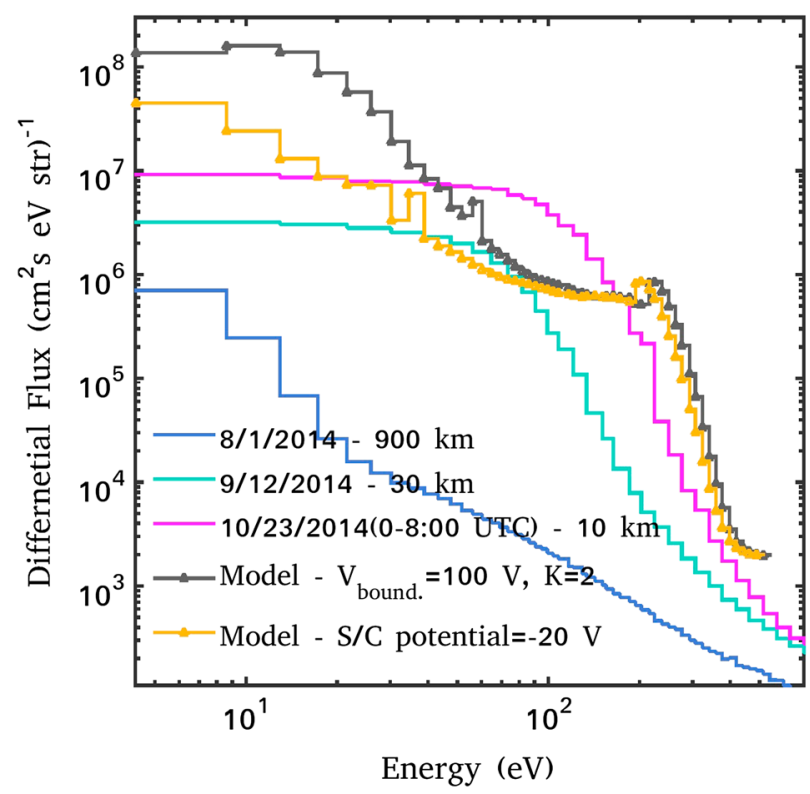

Figure 14. Daily averaged differential electron fluxes versus energy measured by Rosetta IES at distances of $10 \mathrm{~km}$ (red curve), $30 \mathrm{~km}$ (light blue), and $970 \mathrm{~km}$ (blue) from the nucleus are shown. The 1 August spectrum appears to be a solar wind electron distribution. The other two spectra near the nucleus show greatly enhanced fluxes, and the flux dropoffs are at energies of about 100-200 eV. The spectrum on 23 October (00:00-08:00 UTC) is during a CME event. The heliocentric distance of the comet for these observations was about $3 \mathrm{AU}$. Two model spectra are shown for comparison. One is the spectrum for a $100 \mathrm{~V}$ ambipolar potential plus compression from Figure 10. The other (yellow curve) estimates the effects of a $-20 \mathrm{~V}$ spacecraft spectrum by shifting the other model spectrum by $20 \mathrm{eV}$.
Figure 12 shows model spectra for a cometocentric distance of $10 \mathrm{~km}$ and cases (1) and (2). The lowest fluxes are for coma photoelectrons plus solar wind electrons (case 2) without any additional effects. Two gas production rates were adopted: $Q=10^{26} \mathrm{~s}^{-1}$ at $3 \mathrm{AU}$, which is typical, and $Q=10^{-27} s^{-1}$ to account for a possible extreme outburst. Fluxes associated with nucleus photoelectrons are larger than coma photoelectron fluxes, but are relevant only for some locations. The detailed structures in the photoelectron energy spectra are due to lines in the solar radiation spectra that produce photoelectrons at specific energies (i.e., photon energy minus the ionization potential). For example, photoelectron spectra in planetary ionospheres have characteristic peaks near $27 \mathrm{eV}$ due to absorption of solar $\mathrm{He}_{\|} 30.4 \mathrm{~nm}$ photons, and such peaks are evident in Figures 12 and 13 . Putting a boundary potential into the two-stream code (i.e., effects of an ambipolar electric field) enhances the electron fluxes (case 3 ) as shown in Figure 13.

For case (3), the effects of the ambipolar/polarization electric field were approximated using a reflecting boundary condition in the two-stream code for energies less than $E_{\mathrm{bound}}=e \cdot \Delta V_{\mathrm{pol}}$. An accelerated solar wind electron spectrum was used as the boundary flux for higher energies $E>E_{\text {bound. }}$ That is, the entire electric field structure in the coma was collapsed into a sharp potential change at the boundary of our model. Several values of $E_{\mathrm{bound}}$ were tried including $E_{\text {bound }}=0 \mathrm{eV}, 50 \mathrm{eV}$, and $100 \mathrm{eV}$ (Figure 13 shows the $100 \mathrm{eV}$ case).

We next tried to model a case (case 4) for which an electron fluid parcel (with its associated frozen-in magnetic field) is slowed down over a distance of the order of the density structure $(\approx 100 \mathrm{~km}$ or less). Note that the magnetic field is not frozen into the ion flow at these length scales but probably is frozen into the electron gas. For a decreasing electron bulk flow velocity, the magnetic field should be enhanced due to the $\mathbf{u}_{e} \times \mathbf{B}$ term in equation (9) plus Faraday's law. In the case of the solar wind interaction with comet 67P, multifluid and hybrid simulations [Rubin et al., 2014] indeed show that a modest increase of the magnetic field is present near the nucleus (i.e., a factor of 2-3). This results in an increase of the electron perpendicular kinetic energy via the conservation of the first adiabatic invariant, $\mu=K_{\text {perp }} / B$ where $K_{\text {perp }}$ is the perpendicular kinetic energy. For this paper, we simply adopt a three-dimensional adiabatic compression of a fluid parcel by a factor of $k \approx 2$ to see how the electron distribution function might be altered. The new electron differential flux relative to the uncompressed flux provided by the two-stream code is given by

$$
F^{\prime}\left(E^{\prime}\right)=k \times F(E)
$$

where the new energies are denoted $E^{\prime}$ with $E=E^{\prime} / k$ and the original differential electron flux is denoted by $F(E)$. Figure 13 shows this case. The peaks in Figure 13 at high energies are due to solar wind electrons accelerated inward by the adopted ambipolar electron potential.

Now we compare some of the simple model spectra with IES data. Figure 14 shows three-omnidirectional electron spectra measured by IES plus one model spectrum (case 4) from Figure 13 but with the energy 


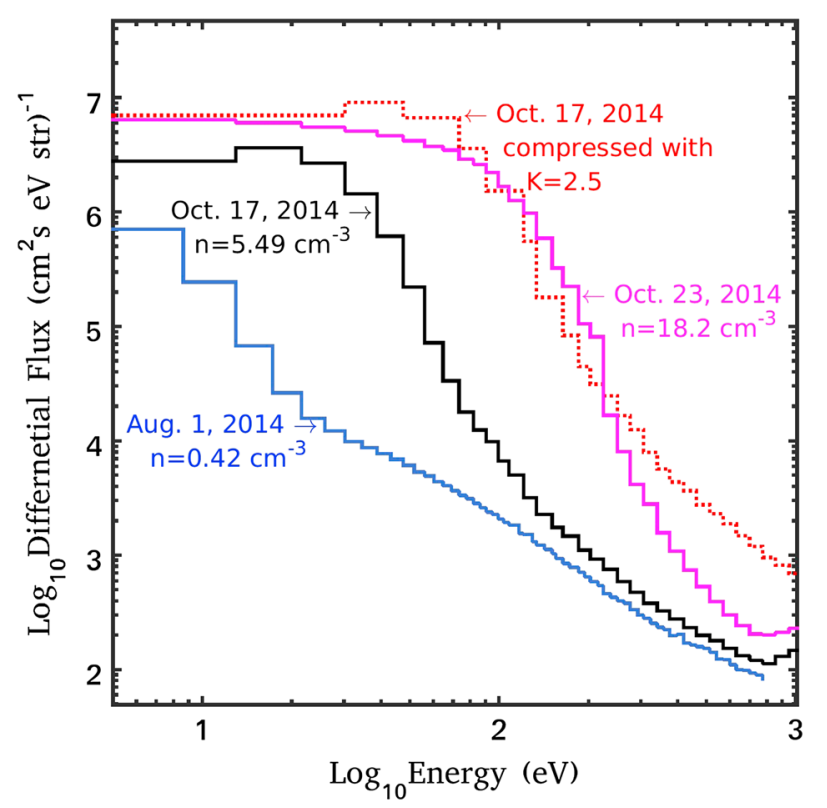

Figure 15. Daily average differential electron fluxes versus energy measured by Rosetta IES at distances of $10 \mathrm{~km}$ (magenta and black curves), and $970 \mathrm{~km}$ (blue) from the nucleus are shown. The 1 August spectrum appears to be a solar wind electron distribution. An interplanetary shock passed the comet on 23 October. The two 17 October spectra differ in that the black curve is just the preshock IES daily average spectrum on 17 October, while its enhanced spectrum with a factor of 2.5 compression, as described in the text, is shown in dotted red. The compressed spectrum is consistent with the extra enhancement associated with a shock passage.

above $50 \mathrm{eV}$. Something is probably still missing from the model for some cases (wave-particle heating perhaps). The underestimation of the electron population in the model spectrum at some energies may also be due to the use of a solar minimum radiation flux. Using a flux for higher solar activity would increase the electron densities and can bring the model results closer to IES. However, even these simple models illustrate how an electron distribution hotter and denser than the $3 \mathrm{AU}$ solar wind distribution can be created.

Some support for compression effects being important is shown in Figure 15, which shows three IES spectra plus a modified IES spectrum. An interplanetary shock passed the comet between the 22 October 16:30 UT and the 23 October 08:00 UT measurements. The measured electron fluxes were significantly enhanced in this time interval. To see if some extra compression (via shock-related effects) could help explain this, we simply took the preshock IES spectrum on 17 October and transformed it using equation (10) with a factor of 2.5. This appears to work well in explaining the compression being a factor in observed enhanced electron fluxes. Edberg et al. [2016] studied four CME/CIR events between October 2014 to December 2014, including the event discussed here, and concluded that the enhancement in densities is due to plasma compression as well as other possible effects such as formation of a plasma boundary or tail disconnection events.

\section{Discussion of Plasma and Field Conditions Near the Comet}

\subsection{Plasma Conditions Near Comet 67P for Autumn 2014 and 3 AU}

Rosetta measurements have shown that the plasma environment near the nucleus of comet 67P at $3 \mathrm{AU}$ is very different from that of active comets, such as Halley in 1986. A standard MHD shock, such as found upstream of the Earth's magnetopause or upstream of a very active comet like Halley, is not present. The creation of a dense plasma (electrons and ions) cloud near the nucleus with a size scale much smaller than an ion gyroradius or ion inertial length affects electrons much more than solar wind ions. Newly created photoions and photoelectrons have low bulk flow velocities with respect to the nucleus (and neutral gas). However, the transition from flowing solar wind electron gas to slow photoelectron gas over some tens of kilometers could be shock-like but not an MHD shock [Cravens, 1997]. 
Table 2. Typical Conditions Near the Nucleus of Comet 67P at $3 \mathrm{AU}$ (From Rosetta Data and/or Models ${ }^{\mathrm{a}}$ )

\begin{tabular}{lc}
\hline Cometocentric distance $(r)$ & $30 \mathrm{~km}(10-100 \mathrm{~km})$ \\
Neutral density $\left(n_{n}\right)$ & $10^{7} \mathrm{~cm}^{-3}\left(10^{6}-10^{8} \mathrm{~cm}^{-3}\right)$ \\
Electron density $\left(n_{e}=n_{i}\right)$ & $20 \mathrm{~cm}^{-3}\left(10-100 \mathrm{~cm}^{-3}\right)$ \\
Average electron energy $\left(k T_{\text {eff }}\right)$ & $20 \mathrm{eV}(10-200 \mathrm{eV})$ \\
Peak magnetic field $\left(B_{\text {max }}\right)$ & $5 \mathrm{nT}^{\mathrm{a}}$ \\
Local electron gyroradius $\left(r_{\text {ecom }}\right)$ & $5 \mathrm{~km}^{\mathrm{a}}$ \\
Charge exchange mean free path $\left(\lambda_{\text {mfpcx }}\right)$ & $1000 \mathrm{~km}\left(10-10^{4} \mathrm{~km}\right)$ \\
Electron neutral mean free path $\left(\lambda_{\mathrm{mfpen}}\right)$ & $10^{4} \mathrm{~km}$ \\
Debye length & $5 \mathrm{~m}$ \\
Electron inertial length $\left(c / \omega_{e}\right)$ & $300 \mathrm{~m}$ \\
lon inertial length $\left(c / \omega_{\text {pi }}\right)$ & $10 \mathrm{~km}$ \\
Solar wind electric field $\left(E_{\mathrm{sw}} \approx u_{\mathrm{sw}} B_{\mathrm{sw}}\right)$ & $5 \times 10^{-4} \mathrm{~V} / \mathrm{m}$ \\
Potential difference: & $20 \mathrm{~V}(10-100 \mathrm{~V})$ \\
$\quad$ Across inner coma $\left(\Delta V \approx r E_{\mathrm{sw}}\right)$ & $10-200 \mathrm{~V}$ \\
$\quad$ Empirical (IES) $\Delta V$ & $4 \mathrm{~km} / \mathrm{s}(\mathrm{at} 50 \mathrm{~km})$ \\
Cometary ion speed $\left(u_{i}=\sqrt{\frac{E_{\mathrm{sw}} r e}{m_{i}}}\right)^{\mathrm{b}}$ & $5000 \mathrm{~km} / \mathrm{s}$ \\
Average "thermal" electron speed $\left(v_{\text {the }}\right)$ & $20 \mathrm{~s}(1-50 \mathrm{~s})$ \\
Cometary ion transport time $\left(\tau_{\text {trans }} \approx r / u_{\mathrm{i}}\right)$ & $10^{-2} \mathrm{~s}$ \\
Electron transport time $\left(\tau_{\text {etrans }} \approx r / v_{\text {the }}\right)$ &
\end{tabular}

${ }^{a}$ E.g., Rubin et al. [2014]. Possible ranges of parameters are shown in parentheses.

${ }^{\mathrm{b}}$ Note $m_{i}=18 m_{p}$ where $m_{p}$ is the proton mass.
Table 2 provides estimates of relevant parameters for the cometary environment, based on Rosetta data. Also, consider the schematic (Figure 11b). Solar wind ion scales $\left(10^{4} \mathrm{~km}\right)$ greatly exceed the density structure size scale, which is roughly the same as Rosetta's distance from the nucleus $(10-100 \mathrm{~km})$ during the autumn of 2014. Note that the photoionization length scale for neutrals is about $10^{7} \mathrm{~km}$. And the ion inertial length (about $50-100 \mathrm{~km}$ ) is of the order of the cometocentric distance of Rosetta. lons behave kinetically near the nucleus. Ion gyroradii greatly exceed the distance scale (i.e., cometocentric distance $r$ ) such that single-fluid MHD theory does not apply. Hence, concepts used in MHD theory (e.g., pressure balance) have to be approached carefully. The electron gyroradius of about $10 \mathrm{~km}$ is somewhat less than the enhancement scale size $(100 \mathrm{~km})$, suggesting that the electrons perhaps can be treated approximately as a fluid. However, this does not mean that the electron distribution function must be Maxwellian, and IES data shows that the distribution function is definitely not Maxwellian. However, pressure as the second velocity moment of the distribution function $\left(p_{e}=n_{e}<\mathrm{KE}>\right.$ ) should remain a useful dynamical quantity and the generalized Ohm's law (equation (9)) should be valid, thus ensuring quasi-neutrality.

The Debye length in the solar wind and/or near the nucleus is only a few meters. Other possibly useful kinetic length scales are the electron and ion inertial lengths of $300 \mathrm{~m}$ and $10 \mathrm{~km}$, respectively. Cometary ion speeds due to acceleration by the motional electric field are about $20 \mathrm{~km} / \mathrm{s}$-much less than the solar wind speed or the electron thermal velocity.

\subsection{Electron Impact Ionization Near Comet 67P}

Another complicating effect in an already complicated scenario is electron impact ionization of neutrals. We calculated electron impact ionization rates by means of a simple integration over energy of measured differential electron fluxes (e.g., Figure 14) times electron impact ionization cross sections for $\mathrm{H}_{2} \mathrm{O}$ and $\mathrm{CO}_{2}$ [cf. Cravens et al., 1987]. The electron impact ionization rate (or ion production rate) divided by the neutral density gives an ionization frequency, as listed in Table 3. We carried this out for several IES electron spectra (as listed) including two shown in Figure 14. In the unperturbed solar wind at $3 \mathrm{AU}$, far from the nucleus, the

Table 3. $\mathrm{CO}_{2}$ and $\mathrm{H}_{2} \mathrm{O}$ Electron Impact Ionization Frequency for IES Electron Spectra Near Comet 67P (Differential Flux Spectra Are Shown in Figure 3)

\begin{tabular}{lcccc} 
Date & Heliocentric Distance $(\mathrm{AU})$ & Cometocentric Distance $(\mathrm{km})$ & $l^{\mathrm{a}}\left(\mathrm{CO}_{2}\right)$ & $I^{\mathrm{a}}\left(\mathrm{H}_{2} \mathrm{O}\right)$ \\
\hline $8 / 1 / 2014$ & 3.6 & 970 & 0.019 & 0.013 \\
$10 / 2 / 2014$ & 3.3 & 10 & 2.9 & 2.41 \\
$10 / 23 / 2014$ & 3.1 & 9.8 & 9.69 & 6.6 \\
$11 / 5 / 2014$ & 3.0 & 31 & 2.6 & 1.95 \\
$2 / 2 / 2015$ & 2.4 & 28 & 2.19 & 1.57 \\
$3 / 23 / 2015$ & 2.0 & 80 & 2.32 & 1.69 \\
$6 / 5 / 2015$ & 1.5 & 205 & 0.59 & 0.44 \\
\hline
\end{tabular}

${ }^{\mathrm{a}}$ Units of $l: 10^{-7} \mathrm{~s}^{-1}$; total ion production rate divided by neutral density. 
electron impact ionization frequency is $I=1.3 \times 10^{-9} \mathrm{~s}^{-1}$ (for water and the solar wind electron spectrum shown in Figure 3). Near the nucleus values ranging from $I=1$ to $9 \times 10^{-7} \mathrm{~s}^{-1}$ were obtained. For comparison, the combined photoionization (solar radiation) and solar wind proton charge exchange cometary ion creation frequency (i.e., production rate divided by neutral density) used in our test particle ion model was $I=10^{-7} \mathrm{~s}^{-1}$.

The higher ionization frequencies should have the most effect on the solar wind interaction with the comet near the nucleus and at larger heliocentric distances (as shown in Table 3) where the contribution of photoionization due to solar radiation is less. The overall effect of increased ionization would be to increase the local density of cometary ions beyond what photoionization would give, and this would enhance the overall interaction. Detailed models of the interaction should include in the future this new ionization. Our electron two-stream code automatically includes electron impact ionization but the ion test particle code did not. The pickup ion density structure will be enhanced by electron impact ionization just discussed.

\subsection{Interpretation and Discussion}

One of the interesting features of the electron data (e.g., Figures $5 \mathrm{~b}$ and 6 ) is that close to the nucleus (within $\sim 100 \mathrm{~km})$, a cold $(E \leq 4 \mathrm{eV})$ thermal electron population exists in addition to a suprathermal population. At larger cometocentric distances this cold population does not appear to be present or is very scarce. A cold "ionospheric" electron population was present in the inner coma (within cometocentric distances of a few thousand kilometers) of comet Halley during its perihelion and was attributed to high electron neutral cooling rates [Gan and Cravens, 1990; Gombosi et al., 1996]. However, the gas production rate of comet 67P near $3 \mathrm{AU}$ was more than 1000 times less than comet Halley's and the presence of cold electrons is perhaps more surprising. In this section, we estimate some electron time constants and find that the existence of a small $(r<100 \mathrm{~km})$ region containing cold electrons is indeed reasonable.

The electrons in the cometary environment are initially created as suprathermal electrons-photoelectrons from photoionization by solar EUV and soft X-ray radiation or solar wind electrons that travel into the inner coma. These $5-50 \mathrm{eV}$ electrons have speeds of about $1000 \mathrm{~km} / \mathrm{s}$ or greater and can traverse the $\sim 30 \mathrm{~km}$ inner coma in about $\tau_{\text {trans }}=\frac{r}{V_{e}} \approx 0.03 \mathrm{~s}$. In addition, the electron-neutral collision mean path greatly exceeds $30 \mathrm{~km}$. If the electrons were free to travel, then the initial fluxes and densities would remain relatively unaltered (and suprathermal) as illustrated by the two-stream model results shown in Figure 13 when no ambipolar electric field was included. Another way to show this is that the cooling time associated with electron-neutral collisions even in the inner coma at $10-30 \mathrm{~km}$ greatly exceeds the electron transit time. The electron water cooling rate for marginally suprathermal electrons of about 1-5eV, dominated by rotational and vibrational collisions, is about $L \approx 10^{-8} \mathrm{eV} \mathrm{cm}^{3} \mathrm{~s}^{-1}$ [cf. Gan and Cravens, 1990; Cravens and Korosmezey, 1986; Cravens, 1989; Schunk and Nagy, 2009], giving a cooling time of $\tau_{\mathrm{en}}=\frac{k T_{e}}{L n_{n}} \approx 50 \mathrm{~s}$ with $k T_{e}=5 \mathrm{eV}$ and $n_{n} \approx 10^{7} \mathrm{~cm}^{-3}$ at $30 \mathrm{~km}$ and only about $10 \mathrm{~s}$ at $10 \mathrm{~km}$. At $100 \mathrm{~km}, \tau_{\mathrm{en}} \approx 500 \mathrm{~s}$. In any case, $\tau_{\mathrm{en}}$ greatly exceeds the transit time. However, as discussed earlier in the paper, we know that an ambipolar field that enforces quasi-neutrality must operate to increase the electron density to the larger values associated with the ions in the slower ion motion. The coexistence of slow ions with fast electrons creates an electrical potential that draws electrons into the ion-rich coma so that the charge quasi-neutrality can be attained.

The residence time of the plasma (ions and electrons) in the inner coma is the length scale $r$, divided by the average ion flow speed $u_{i} \approx 1-10 \mathrm{~km} / \mathrm{s}$, and is largely associated with the newborn ions accelerated by the electric field (motional plus ambipolar). The residence time for the ion motion is about $\tau_{\text {res }}=\frac{r}{u_{i}} \approx 3-30 \mathrm{~s}$, for 10 to $100 \mathrm{~km}$. Figures 9 and 10 showed ion densities from our simple ion pickup model and from global simulations [Rubin et al., 2014]. One can also estimate the residence time from a dimensional analysis of the continuity equation ignoring losses: $\tau_{\text {res }}=\frac{n_{e}}{P}$, where $P$ is the ion-electron production rate which can be written as $P=\ln n_{n} . l \approx 10^{-7} \mathrm{~s}^{-1}$ is the ionization frequency discussed earlier in the paper. In the 10-30 km region, this gives $\tau_{\text {res }} \approx 10-100 \mathrm{~s}$, which is comparable to the cooling time. In other words, the electron population, starting out suprathermal, has time to evolve into a colder population. For $r>100 \mathrm{~km}$ or so, $n_{e} \approx 30 \mathrm{~cm}^{-3}$ and $\tau_{\text {res }} \approx 300 \mathrm{~s}$. But the electron neutral cooling time is becoming even larger, and the electrons will remain suprathermal. Note that the electron-ion dissociative recombination time, $\tau_{\mathrm{DR}} \approx 1 /\left(\alpha \cdot n_{e}\right) \approx 10^{4} \mathrm{~s}$, greatly exceeds all the time scales discussed above. The dissociative recombination rate coefficient is $\alpha \approx 10^{-7}-10^{-6} \mathrm{~cm}^{3} \mathrm{~s}^{-1}$ [Vigren et al., 2013]. 
Another question is whether or not the cold electrons have a Maxwellian distribution function; we know from IES that the suprathermal electrons do not. For very cold electrons (e.g., $E \sim 0.1 \mathrm{eV}$ or $T_{e} \approx 10^{3} \mathrm{~K}$ ) at $10 \mathrm{~km}$ the electron-electron collision frequency is given by $v_{e e}=54.5 \frac{n_{e}}{T_{e}^{1.5}} \approx 0.15 \mathrm{~s}^{-1}$ [Schunk and Nagy, 2009], corresponding to a time constant of $\tau_{e e} \sim 6 \mathrm{~s}$. For somewhat "hotter" cold electrons with $E \sim 1 \mathrm{eV}$ $\left(T_{e} \sim 10^{4} \mathrm{~K}\right), \tau_{e e} \approx 200 \mathrm{~s}$. This shows that cold electrons can experience at least one Coulomb collision while being constrained by the ambipolar electric field. The cold electron population near the nucleus $(r<100 \mathrm{~km})$ can indeed be described as Maxwellian or thermalized.

For comet Halley Gan and Cravens [1990] described an electron thermal "collisionopause" located at a cometocentric distance of about $1-2 \times 10^{4} \mathrm{~km}$, within which a cold electron population existed and beyond which the electron temperature rapidly increased due to higher electron-neutral cooling times. This boundary was manifested as a transition in the observed (and modeled) electron density [Rème et al., 1986; Rème, 1991; Cravens et al., 1987]. Apparently, such a transition also exists for comet 67P at $3 \mathrm{AU}$ but at a distance of only $\approx 100 \mathrm{~km}$.

The observations also support these claims reasonably well. The interplay between the hot and cold electron populations and neutral densities is in fact visible in Figure $5 \mathrm{~b}$ where there is an anticorrelation between IES electron and LAP ion densities. Edberg et al. [2015] showed a correlation between the LAP/MIP peak densities and the neutral density variations. Such correlation fits well with the discussion given here on the electronneutral collisional cooling process of hot electrons. The LAP and IES electron densities shown in Figure 6 also suggest that the cold electron population becomes significant within $100 \mathrm{~km}$ from the comet. At a distance of $50 \mathrm{~km}$, an average density of about $40 \mathrm{~cm}^{-3}$ can be estimated for the cold thermal electrons and much higher densities at closer distances. It must be noted that the uncertainty of our methodology in calculating the IES electron density, which can be a factor of 2-3 change in the derived densities, does not obscure the given interpretations and the relation between the population of thermal and suprathermal electrons remains the same.

\section{Conclusion}

In this paper we investigated the behavior of pickup ions and electrons near the nucleus of comet 67P using Rosetta IES data plus model calculations. The Rosetta IES experiment revealed the presence of greatly enhanced electron fluxes and densities near the nucleus, confirmed by the density measurements of the LAP and MIP instruments. Our modeling efforts demonstrated that the observed electrons are a combination of compressed solar wind electrons plus photoelectrons due to the photoionization of the comet's surface and the coma gas by solar radiation. The electron density and flux enhancements that were observed are evidently associated with an ion density enhancement consisting of slowly moving pickup ions created by the ionization of neutrals. Such a density enhancement was predicted by pre-encounter simulations [Rubin et al., 2014]. We also conclude that unperturbed photoelectron fluxes or solar wind electron fluxes at some energies are too low to match measured fluxes. Each photoion (i.e., pickup ion) created comes with a photoelectron, but the electron fluxes (and densities) associated with the photoelectrons in the two-stream model are not sufficient to explain the IES data in the range of 5-200 eV (see Figure 14), while the lower energy electrons are much higher than the observations. The ions move slowly so that their density builds up, whereas the electrons quickly escape from the vicinity of the nucleus (i.e., compare the time scales in Table 2 for electron and ion transport), unless they are prevented by electric fields or collisions.

Three processes must be operating near the nucleus: (1) collisional cooling of suprathermal electrons through electron-neutral collisions, (2) confinement of the electrons near the nucleus by an ambipolar electric field, and (3) enhancement of the fluxes due to compression of the electron gas near the nucleus. We also showed that at $3 \mathrm{AU}$ within $100 \mathrm{~km}$ from the nucleus, cooling of suprathermal electrons through electron-neutral collisions becomes significant and a population of cold thermal electrons begins to build up, which is also confined by the ambipolar electric field.

Compression due to deceleration, confinement by an ambipolar field, and addition of electrons by photoionization, all evidently play a role in determining the electron distribution function (i.e., electron spectrum), but our models put these effects in sequentially and simplistically rather than simultaneously. Nonetheless, the model results and the IES electron data agree in many respects, indicating that we have probably 
identified key processes in the near-nucleus solar wind-comet interaction at $3 \mathrm{AU}$; but many details remain unexplained due to the complexity and time variability of this plasma environment.

Electron impact processes have been known for a long time to affect airglow emission from comets [e.g., Cravens and Green, 1978; Feldman et al., 2004]. The enhanced electron fluxes (from the solar wind and/or photoelectrons) considered in the current paper could explain the enhanced $\mathrm{H}_{1}, \mathrm{O}_{1}$, and $\mathrm{C}_{1}$ emissions from dissociation of $\mathrm{H}_{2} \mathrm{O}$ and $\mathrm{CO}_{2}$ observed by the Alice (Rosetta's UV imaging spectrograph) instrument, as discussed by Feldman et al. [2015]. This would make a good future project.

\section{Acknowledgments}

All data shown in the figures can be obtained from the corresponding author. The work at the University of Kansas has been supported by NASA's Planetary Atmospheres program, grant NNX14AG79G (model development) and by the Rosetta project through a subcontract from the Southwest Research Institute. Rosetta is an ESA mission with contributions from its member states and NASA. We also thank AMDA@ for providing the Rosetta-CG distance data. The work on RPC-LAP data was funded by the Swedish National Space Board under contracts $109 / 02$ and $135 / 13$ and the Swedish Research Council under contract 621-2013-4191.

\section{References}

Balsiger, H., et al. (1986), lon composition and dynamics at Comet Halley, Nature, 321, 330-334.

Balsiger, H., et al. (2007), ROSINA: Rosetta Orbiter Spectrometer for Ion and Neutral Analysis, Space Sci. Rev., 128, $745-801$.

Bieler, A., et al. (2015), Comparison of 3D kinetic and hydrodynamic models to ROSINA-COPS measurements of the neutral coma of 67P/Churyumov-Gerasimenko, Astron. Astrophys., 583, A7, doi:10.1051/0004-6361/201526178.

Broiles, T. W., J. L. Burch, G. B. Clark, C. Koenders, E. Behar, R. Goldstein, S. A. Fuselier, K. E. Mandt, P. Mokashi, and M. Samara (2015), Rosetta observations of solar wind interaction with the comet 67P/Churyumov-Gerasimenko, Astron. Astrophys., 583, doi:10.1051/0004-6361/ 201526046.

Burch, J. L., R. Goldstein, T. E. Cravens, W. C. Gibson, R. N. Lundin, C. J. Pollock, J. D. Winningham, and D. T. Young (2007), RPC-IES: The ion and electron sensor of the Rosetta Plasma Consortium, Space Sci. Rev., 128, 697-712.

Burch, J. L., T. E. Cravens, K. Llera, R. Goldstein, P. Mokashi, C.-Y. Tzou, and T. Broiles (2015), Charge exchange in cometary coma: Discovery of $\mathrm{H}^{-}$ions in the solar wind close to comet 67P/Churyumov-Gerasimenko, Geophys. Res. Lett., 42, 5125-5131, doi:10.1002/ 2015GL064504.

Carr, C., et al. (2007), RPC: The Rosetta Plasma Consortium, Space Sci. Rev., 128, 629-647, doi:10.1007/s11214-006-9136-4.

Clark, G., et al. (2015), Suprathermal electron environment of comet 67P/Churyumov-Gerasimenko: Observations from the Rosetta lon and Electron Sensor, Astron. Astrophys., 583, A24, doi:10.1051/0004-6361/201526351.

Coates, A. J., A. D. Johnston, B. Wilken, K. Jockers, and K.-H. Glassmeier (1989), Velocity space diffusion of pickup ions from the water group at comet Halley, J. Geophys. Res., 94, 9983-9993, doi:10.1029/JA094iA08p09983.

Cravens, T. E. (1986), Ion distribution functions in the vicinity of Comet Giacobini-Zinner, Geophys. Res. Lett., 13, 276-278, doi:10.1029/ GL013i003p00275.

Cravens, T. E. (1989), Test particle calculations of pick-up ions in the vicinity of comet Giacobini-Zinner, Planet. Space Sci., 37, 1169-1184

Cravens, T. E. (1997), Physics of Solar System Plasmas, Cambridge Univ. Press, Cambridge, U. K.

Cravens, T. E., and T. I. Gombosi (2004), Cometary magnetospheres: A tutorial, Adv. Space Sci., 33, 1968-1976.

Cravens, T. E., and A. E. S. Green (1978), Airglow from the inner comas of comets, Icarus, 33, 612-623.

Cravens, T. E., and A. Korosmezey (1986), Vibrational and rotational cooling of electrons by water vapor, Planet. Space Sci., 34, 961-970.

Cravens, T. E., J. U. Kozyra, A. F. Nagy, T. I. Gombosi, and M. Kurtz (1987), Electron impact ionization in the vicinity of comets, J. Geophys. Res., 92, 7341-7353, doi:10.1029/JA092iA07p07341.

Edberg, N. J. T., et al. (2015), Spatial distribution of low-energy plasma around comet 67P/CG from Rosetta measurements, Geophys. Res. Lett., 42, 4263-4269, doi:10.1002/2015GL064233.

Edberg, N. J. T., et al. (2016), Solar wind interaction with comet 67P: Impacts of corotating interaction regions, J. Geophys. Res. Space Physics, 121, 949-965, doi:10.1002/2015JA022147.

Eriksson, A. I., et al. (2007), RPC-LAP: The Rosetta langmuir probe instrument, Space Sci. Rev., 128, 729-744, doi:10.1007/s11214-006-9003-3. Feldman, P. D., A. L. Cochran, and M. R. Combi (2004), Spectroscopic investigations of fragment species in the coma, in Comets II, edited by M. C. Festou, H. U. Keller, and H. A. Weaver, pp. 425-447, Univ. of Arizona Press, Tucson.

Feldman, P. D., et al. (2015), Measurements of the near-nucleus coma of comet 67P/Churyumov-Gerasimenko with the Alice far-ultraviolet spectrograph on Rosetta, Astron. Astrophys., 583, A8, doi:10.1051/0004-6361/201525925.

Galeev, A. A., T. E. Cravens, and T. I. Gombosi (1985), Solar wind stagnation near comets, Astrophys. J., 289, 807-819.

Gan, L., and T. E. Cravens (1990), Electron energetics in the inner coma of comet Halley, J. Geophys. Res., 95, 6285-6303, doi:10.1029/ JA095iA05p06285.

Glassmeier, K.-H., I. Richter, A. Diedrich, G. Musmann, U. Auster, U. Motschmann, and A. Balogh (2007), RPC-MAG the fluxgate magnetometer in the Rosetta Plasma Consortium, Space Sci. Rev., 128, 649-670, doi:10.1007/s11214-006-9114-x.

Gloeckler, G., et al. (1986), Cometary pick-up ions observed near Giacobini-Zinner, in Comet Encounters, edited by T. Birmingham and A. Dessler, AGU, Washington, D. C., doi:10.1029/SP027p0028.

Goldstein, R., et al. (2015), The Rosetta Ion and Electron Sensor (IES) measurement of the development of pickup ions from comet 67P/Churyumov-Gerasimenko, Geophys. Res. Lett., 42, 3093-3099, doi:10.1002/2015GL063939.

Gombosi, T. I. (2015), Physics of cometary magnetospheres, in Magnetotails in the Solar System, edited by A. Keiling, C. M. Jackman, and P. A. Delamere, John Wiley, Hoboken, N. J., doi:10.1002/9781118842324.ch10.

Gombosi, T. I., D. L. De Zeeuw, R. M. Häberli, and K. G. Powell (1996), Three dimensional multiscale MHD model of cometary plasma environment, J. Geophys. Res., 101(A7), 15,233-15,253, doi:10.1029/96JA01075.

Gulkis, S., et al. (2015), Subsurface properties and early activity of Comet 67P/Churyumov-Gerasimenko, Science, 347(6220), doi:10.1126/ science.aaa0276.

Hansen, K. C., C. Alexander, T. Bagdonat, M. R. Combi, T. E. Cravens, T. I. Gombosi, Y.-D. Jia, U. Motschmann, and I. P. Robertson (2007), The plasma environment of Comet 67P/Churyumov-Gerasimenko throughout the Rosetta Main Mission, Space Sci. Rev., 128, 133-166, doi:10.1007/s11214-006-9142-6.

Haser, L. (1957), Distribution d'intensité dans la têd' une comète, Bull. Acad. R. d'Belgique Classe Sci., 34(5), 740-750.

Hassig, M., et al. (2015), Time variability and heterogeneity in the coma of Comet 67P/ Churyumov-Gerasimenko, Science, 347(6220), doi:10.1126/science.aaa0276.

Hsu, H.-W., M. Horányi, S. Kempf, and E. Grün (2012), Spacecraft charging near Enceladus, Geophys. Res. Lett., 39, L06108, doi:10.1029/ 2012GL050999. 
Jia, Y. D., M. R. Combi, K. C. Hansen, T. I. Gombosi, F. J. Crary, and D. T. Young (2008), A 3-D global MHD model for the effect of neutral jets during the Deep Space 1 Comet 19P/Borrelly flyby, Icarus, 196, 249-257.

Johnstone, A. D., A. J. Coates, D. E. Huddleston, K. Jockers, B. Wilken, and H. Borg (1993), Observations of the solar wind and cometary ions in the encounter between Giotto and Comet Grigg-Skjellerup, Astron. Astrophys., 273, L1-L4.

Khazanov, G. V. (2011), Kinetic theory of the inner magnetospheric plasma, Astrophys. Space Sci. Lib., 372, doi:10.1007/978-1-4419-6797-8_2.

Koenders, C., K. H. Glassmeier, I. Richter, U. Motschmann, and M. Rubin (2013), Revisiting cometary bow shock positions, Planet. Space Sci., 87, 85-95.

Koenders, C., K. H. Glassmeier, I. Richter, H. Ranocha, and U. Motschmann (2015), Dynamical features and spatial structures of the plasma interaction region of 67P/Churyumov-Gerasimenko and the solar wind, Planet. Space Sci., 105, 101-116, doi:10.1016/j.pss.2014.11.014.

Li, Y., P. H. Yoon, C. S. Wu, A. T. Weatherwax, J. K. Chao, and B. H. Wu (1997), lon pitch-angle scattering by Alfvén waves, Phys. Plasmas, 4, 4103-4117, doi:10.1063/1.872530.

Neugebauer, M., et al. (1987), The pickup of cometary protons by the solar wind, Astron. Astrophys., 187, 21-24.

Nilsson, H., et al. (2007), RPC-ICA: The ion composition analyzer of the Rosetta Plasma Consortium, Space Sci. Rev., 128, 671-695, doi:10.1007/ s11214-006-9031-z.

Nilsson, H., et al. (2015), Birth of a comet magnetosphere: A spring of water ions, Science, 347, 0571, doi:10.1126/science.aaa0571.

Odelstad, E., A. I. Eriksson, N. J. T. Edberg, F. Johansson, E. Vigren, M. André, C.-Y. Tzou, C. Carr, and E. Cupido (2015), Evolution of the plasma environment of comet 67P from spacecraft potential measurements by the Rosetta Langmuir probe instrument, Geophys. Res. Lett., 42, 10,126-10,134, doi:10.1002/2015GL066599.

Rahmati, A., T. E. Cravens, A. F. Nagy, J. L. Fox, S. W. Bougher, R. J. Lillis, S. A. Ledvina, D. E. Larson, P. Dunn, and J. A. Croxell (2014), Pickup ion measurements by MAVEN: A diagnostic of photochemical oxygen escape from Mars, Geophys. Res. Lett., 41, 4812-4818, doi:10.1002/ 2014 GL060289.

Rahmati, A., D. E. Larson, T. E. Cravens, R. J. Lillis, P. A. Dunn, J. S. Halekas, J. E. Connerney, F. G. Eparvier, E. M. B. Thiemann, and B. M. Jakosky (2015), MAVEN insights into oxygen pickup ions at Mars, Geophys. Res. Lett., 42, 8870-8876, doi:10.1002/2015GL065262.

Rème, H. (1991), Cometary plasma observations between the shock and the contact surface, in Cometary Plasma Processes, AGU Geophysical Monograph Series, vol. 61, edited by A. D. Johnstone, pp. 87-105, AGU, Washington D. C., doi:10.1029/GM061p0087.

Rème, H., et al. (1986), Comet Halley-solar wind interaction from electron measurements aboard Giotto, Nature, 321, 349-352, doi:10.1038/ 321349a0.

Richard, M., et al. (2015), An empirical approach to modeling ion production rates in Titan's ionosphere I: Ion production rates on the dayside and globally, J. Geophys. Res. Space Physics, 120, 1264-1280, doi:10.1002/2013JA019706.

Rubin, M., et al. (2014), Plasma environment of a weak comet-Predictions for Comet 67P/ Churyumov-Gerasimenko from multifluid MHD and hybrid models, Icarus, 242, 38-49, doi:10.1016/j.icarus.2014.07.021.

Schunk, R. W., and A. F. Nagy (2009), lonospheres, 2nd ed., Cambridge Univ. Press, Cambridge, U. K.

Trotignon, J. G., et al. (2007), RPC-MIP: The mutual impedance probe of the Rosetta plasma consortium, Space Sci. Rev., 128, 713-728, doi:10.1007/s11214-006-9005-1.

Vigren, E., et al. (2013), Predictions of ion production rates and ion number densities within the diamagnetic cavity of comet 67P/Churyumov-Gerasimenko at perihelion, Astrophys. J., 772, 33, doi:10.1088/0004-637X/772/1/33.

Vigren, E., M. Galand, A. I. Eriksson, N. J. T. Edberg, E. Odelstad, and S. J. Schwartz (2015), On the electron-to-neutral number density ratio in the coma of comet 67P/Churymov-Gerasimenko: Guiding expression and sources for deviations, Astrophys. J., 812(1), 54, doi:10.1088/ 0004-637X/812/1/54.

Weingartner, J. C., and B. T. Draine (2001), Photoelectric emission from interstellar dust: Grain charging and gas heating, Astrophys. J. Suppl., $134,263-282$.

Wiehle, S., U. Motschmann, N. Gortsas, K.-H. Glassmeier, J. Müller, and C. Koenders (2011), Simulation of cometary jets in interaction with the solar wind, Adv. Space Res., 48, 1108-1113. 